\title{
A Formula for the Colored Jones Polynomial of 2-Bridge Knots
}

\author{
TOShie TAKata \\ Department of Mathematics, Faculty of Science, Niigata University, Niigata 950- \\ 2181, Japan \\ e-mail : takata@math.sc.niigata-u.ac.jp
}

Abstract. We derive a formula for the colored Jones polynomial of 2-bridge knots. For a twist knot, a more explicit formula is given and it leads to a relation between the degree of the colored Jones polynomial and the crossing number.

\section{Introduction}

For a positive integer $n$, the $n$-colored Jones polynomial is a Laurent polynomial invariant in $q$ of a knot colored with $n$-dimensional irreducible representation of $U_{q}\left(s l_{2}\right)$. We denote by $J_{K}(n)$ the $n$-colored Jones polynomial of a knot $K$, which is normalized so that the colored Jones polynomial of the trivial knot is 1 . Then, $J_{K}(2)$ coincides with the original Jones polynomial of a knot $K$ defined by $\mathrm{V}$. F. R. Jones [3]. K. Habiro [2] and T. T. Q. Le gave explicit formulas for the $n$-colored Jones polynomials of the trefoil knot and the figure eight knot. Moreover, a formula for the colored Jones polynomials of twist knots was given by G. Masbaum [7], using skein theory.

In this paper, we give a formula for the colored Jones polynomial of the 2-bridge knot $S(p, t)$, using a formula obtained by R. Lawrence and O. Ron in [5]. As an application of our formula, we obtain a relation between the degree of the colored Jones polynomial and the crossing number for the 2 -bridge knots $S(4 p+1,2 p+1)$ and $S(4 p-1,2 p-1)$.

Let $p$ and $t$ be coprime odd integers with $p>t>1$. We denote by $S(p, t)$ the 2-bridge knot of type $(p, t)$ using Schubert notation. We explain how to draw a diagram of the 2-bridge knot $S(p, t)$ (see [1], [4]). Drawn two disjoint vertical segments $w_{1}=A B$ and $w_{2}=C D$, divide each segments into $p$ segments, and number from 0 to $2 p-1$ in both sides of the dividing points of each segment as pictured in Figure 1. The points $B$ and $D$ are numbered 0 , and the points $A$ and $C$ numbered $p$. Next draw two disjoint simple curves $v_{1}$ from $B$ to $C$ and $v_{2}$ from $D$ to $A$, which underpass the segments $w_{1}$ and $w_{2}$. Along the curve $v_{1}$, one starts

Received June 29, 2006.

2000 Mathematics Subject Classification: 57M27.

Key words and phrases: Knots, Jones polynomial.

This research is partially supported by Grant-in-Aid for Scientific Research (C) 
from 0 , i.e. $\mathrm{B}$ of the segments $w_{1}$, and meets $w_{2}$ at $t$, and next meets $w_{1}$ at $2 t$, and then meets $w_{2}$ at $3 t$. This is to be repeated until one reaches $p(=C)$ of $w_{2}$. Similarly, along the curve $v_{2}$, one starts from $0(=D)$ of $w_{2}$, and meets $w_{1}$ at $t$, and next meets $w_{2}$ at $2 t$, and then meets $w_{1}$ at $3 t$. This is to be repeated until one reaches $p(=A)$ of $w_{1}$. Here we take it $\bmod 2 p$. This regular projection is called Schubert's normal form. For example, Schubert's normal form for the $S(5,3)$ (the figure eight knot) is as in Figure 1.

$S(5,3)$

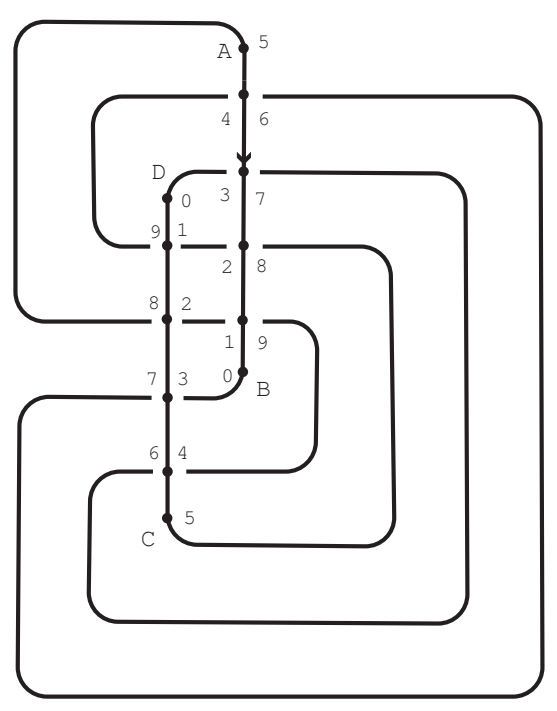

Figure 1.

We will prepare with some notations. Let us put $p^{\prime}=\frac{p-1}{2}$. For $1 \leq j \leq p^{\prime}$, define integers $r(j)$ so that $r(j) \equiv(2 j-1) t \bmod 2 p$ and $-p<r(j)<p$. We put $\sigma_{j}:=\frac{r(j)}{|r(j)|}, r^{\prime}(j):=\frac{|r(j)|+1}{2}$ and $i_{r^{\prime}(j)}=j$. Note that $\sigma_{j}=(-1)^{[(2 j-1) t / p]}$, where $[x]$ denotes the greatest integer less than or equal to $x$. For an integer $i$, $\operatorname{sign}(i)$ is the sign of $i$. We put $\mathbf{m}=\left(m_{1}, \cdots, m_{p^{\prime}}\right), m_{0}=0$. We will also define for $\sigma= \pm 1$

$$
\left(q^{\sigma}\right)_{k}=\left(q^{\sigma}-1\right)\left(\left(q^{\sigma}\right)^{2}-1\right) \cdots\left(\left(q^{\sigma}\right)^{k}-1\right), \quad\left\{\begin{array}{c}
n \\
m
\end{array}\right\}_{\sigma}=\frac{\left(q^{\sigma}\right)_{n}}{\left(q^{\sigma}\right)_{m}\left(q^{\sigma}\right)_{n-m}} .
$$

We obtain

Theorem 1. A formula for the n-colored Jones polynomial of the 2-bridge knot 
$S(p, t)$ is given by

$$
J_{S(p, t)}(n)=\sum_{0 \leq m_{j-1} \leq m_{j} \leq n-1} q^{a(\mathbf{m}) n+b_{1}(\mathbf{m})+b_{2}(\mathbf{m})} X(\mathbf{m}),
$$

where

$$
\begin{aligned}
& a(\mathbf{m})=-\frac{1}{2} \sum_{j=1}^{p^{\prime}}\left(\sum_{k=r^{\prime}(j)}^{p^{\prime}}\left(\sigma_{i_{k}}+\sigma_{i_{p^{\prime}+1-k}}\right)\right)\left(m_{j}-m_{j-1}\right)-\frac{1}{2} \sum_{j=1}^{p^{\prime}-1}\left(\sigma_{j+1}+\sigma_{p^{\prime}+1-j}\right) m_{j} \\
& -\frac{1}{2}\left(\sigma_{p^{\prime}}+1\right) m_{p^{\prime}}-\sum_{j=1}^{p^{\prime}} \sigma_{j} \\
& b_{1}(\mathbf{m})=-a(\mathbf{m}) \\
& +\sum_{1 \leq k \leq \frac{p-t}{2}} \frac{1-\sigma_{i_{k}}}{2} m_{i_{k}-1}-\sum_{\frac{p-t}{2}+1 \leq k \leq p^{\prime}} m_{i_{k}-1}+\sum_{\frac{p-t}{2}+1 \leq k \leq p^{\prime}} \frac{1+\sigma_{i_{k}}}{2} m_{i_{k}} \\
& -\left(1+\sigma_{p^{\prime}}\right) m_{p^{\prime}}+\frac{1}{2} \sum_{j=1}^{p^{\prime}-1}\left(\sigma_{j+1}-\sigma_{j}\right) m_{j} \\
& -\frac{1}{2} \sum_{1 \leq k \leq p^{\prime}-1} \sum_{k+1 \leq k^{\prime} \leq p^{\prime}} \frac{1+\operatorname{sign}\left(i_{k}-i_{k^{\prime}}\right)}{2}\left(\sigma_{i_{k}}-\sigma_{i_{k^{\prime}}}\right)\left(m_{i_{k}}-m_{i_{k}-1}\right)\left(m_{i_{k^{\prime}}}-m_{i_{k^{\prime}}-1}\right) \\
& +\sum_{j=1}^{p^{\prime}} \sigma_{j}\left(\sum_{k=1}^{r^{\prime}(j)}\left(m_{i_{k}}-m_{i_{k}-1}\right)\right) m_{j-1}, \\
& b_{2}(\mathbf{m})=\left\{\begin{array}{lll}
\sum_{\frac{p-t}{2}+1 \leq k \leq \frac{t-1}{2}} \frac{1+\sigma_{i_{k}}}{2^{2}} m_{i_{k}-1} & \text { if } & p<2 t, \\
-\sum_{\frac{t+1}{2}+1 \leq k \leq \frac{p-t}{2}} \frac{1^{2}+\sigma_{i_{k}}}{2} m_{i_{k}-1} & \text { if } & p>2 t,
\end{array}\right. \\
& X(\mathbf{m})=\frac{\left(\bar{q}^{\sigma_{p^{\prime}}}\right)_{n-1}\left(\bar{q}^{\sigma_{p^{\prime}}}\right)_{m_{p^{\prime}}}}{\left(\bar{q}^{\sigma}{ }_{p^{\prime}}\right)_{n-m_{p^{\prime}}-1}}\left(\prod_{j=1}^{p^{\prime}}\left(q^{-\sigma_{j}}\right)_{m_{j}-m_{j-1}}\right)^{-1}(-1)^{m_{p^{\prime}}}
\end{aligned}
$$

The plan of the present paper is as follows. In section 2, we review Lawrence and Ron's result and give a proof for Theorem 1 . In section 3, we get a more explicit formula for the colored Jones polynomial of twist knots and estimate the maximal and minimal degrees.

\section{Proof of Theorem 1}

Firstly, we review a result in [5]. Let $L$ be a knot in $S^{3}$. We consider a 1-tangle $L^{\prime}$ whose closure is $L$. We assume that $L^{\prime}$ is presented as a tangle diagram $D$ in 
generic position with respect to the height function with the end points at the top and at the bottom. We orient $D$ from the top to the bottom. Let $c$ denote the number of the crossings in a diagram $D$. There are eight crossing types given by the orientations on the two strands and the sign of the crossing, two cap types, and two cup types by the orientations on the strand in a tangle diagram. The two crossoing types (1) and (2), the cap type (i), and the cup type (ii) as in Figure 2 are called leftpointing. We construct an enhanced Gauss diagram $G$ for $D$, which is a circle containing the combinatorial data which encodes $D$. We number crossings by $j=1, \cdots, c$ and denote by $\sigma_{j}$ the sign of the $j$-th crossing. We mark the base

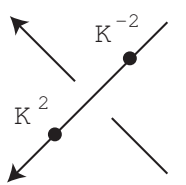

(1)

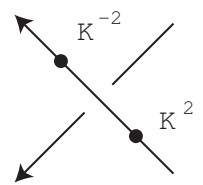

(2)

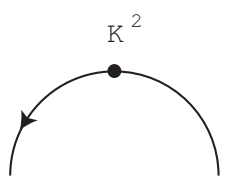

(i)

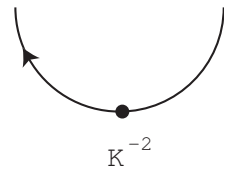

(ii)

Figure 2 .

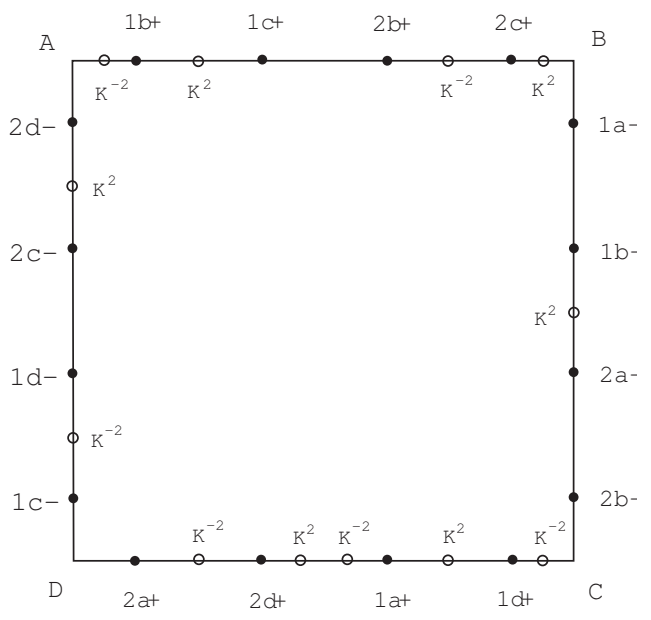

Figure 3.

point on a circle, which corresponds to the end at the top of $D$. As going around the circle clockwise from the base point, with tracing the tangle strand according to its orientation, we mark $2 c$ points indexed by $i=1, \cdots, 2 c$, which correspond to points passing crossings on the tangle strand. So, exactly two points of these $2 c$ points except the base point correspond to one crossing, and one comes from the underpass 
and the other from the overpass. Join these two points on the circle by an oriented chord from overpass to underpass, and assign the sign of the corresponding crossing. Each $i$-th point on the circle is assigned with a pair $\left(j(i), \varepsilon_{i}\right)$, simply denoted by $j(i) \varepsilon_{i}$, where $j(i)$ is the number of the passing crossing, and $\varepsilon_{i}$ is 1 for an overpass and -1 for an underpass. Moreover, the circle is marked with blobs $K^{ \pm 2}$ placed on the leftpointing crossings and cups and caps as in Figure 2. The result is an enhanced Gauss diagram. For example, the enhanced Gauss diagram for Figure 1 is shown in Figure 3, where oriented chords are not drawn. We denote by $i_{1}, \cdots, i_{b}$ the position of the blobs $K^{ \pm 2}$, where the $a$-th blob is placed between the $i_{a}$-th and $\left(i_{a}+1\right)$-th positions on the circle, with $K^{2 \delta_{a}}$.

We put

$$
\begin{aligned}
q_{j}= & \frac{1}{2} \sum_{i>j+} \sigma_{i}-\frac{1}{2} \sum_{i>j-} \sigma_{i}-\frac{1}{2} \sum_{j=1}^{c} \sigma_{j}, \\
d(\mathbf{l})= & \sum_{j=1}^{c} q_{j} l_{j}-\frac{1}{2} \sum_{a} \delta_{a}-\frac{1}{2} \sigma_{j}, \\
C^{0}(\mathbf{l})= & -d(\mathbf{l})-\sum_{\substack{a \\
k \leq i_{a}}} \delta_{a} \varepsilon_{k} l_{k}-\frac{1}{2} \sum_{j} \sigma_{j} l_{j}-\frac{1}{2} \sum_{i} \sigma_{i} \varepsilon_{i} l_{i} \varepsilon_{k} l_{k} \\
& +\sum_{j} \sigma_{j}\left(\sum_{i<j-} \varepsilon_{i} l_{i}\right)\left(\sum_{k<j+} \varepsilon_{k} l_{k}\right) .
\end{aligned}
$$

Theorem 2([5]). A formula for the $n$-colored Jones polynomial of a 0-framed knot $L_{0}$ is given by

$$
=\sum_{\substack{l_{j}=0 \\
j=0, \ldots, c}}^{\infty} q^{C^{n}(\mathbf{l})} \prod_{j=1}^{c}\left\{\begin{array}{c}
s(j)+l_{j} \\
l_{j}
\end{array}\right\}_{\sigma_{j}}\left(q^{\sigma_{j}(n-s(j)-1)}-1\right) \cdots\left(q^{\sigma_{j}\left(n-s(j)-l_{j}\right)}-1\right),
$$

where $s(j)=-\sum_{i<j-} \varepsilon_{i} l_{j(i)}$, and $C^{n}(\mathbf{l})=d(\mathbf{l}) n+C^{0}(\mathbf{l})$. Here $i<j-$ means $i<i_{0}$ for $i_{0}$ such that $j=j\left(i_{0}\right)$ and $\varepsilon_{i_{0}}=-1$.

We apply this formula to the 2-bridge knot $S(p, t)$.

From Schubert's normal form, we get a 1-tangle diagram as in Figure 4 and 5. The corresponding enhanced Gauss diagram without blobs and chords can be expressed in Figure 7. The number of the crossings equals $2(p-1)=4 p^{\prime}$. We name by $j a($ resp. $j d)$ the crossing of the label $(2 j-1) t \bmod 2 p($ resp. $2 j t \bmod 2 p)$ on $C D$ for $1 \leq j \leq p^{\prime}$ and name by $j c$ (resp. $j b$ ) the crossing of the label $(2 j-1) t$ $\bmod 2 p($ resp. $2 j t \bmod 2 p)$ on $A B$ for $1 \leq j \leq p^{\prime}$. For $1 \leq k \leq p^{\prime}$, there exists a unique $1 \leq j \leq p^{\prime}$ such that $2 k-1 \equiv|r(j)| \bmod 2 p$, and then $i_{k} a=j a$ and $i_{k} c=j c$. For $1 \leq j \leq p^{\prime}$, we define integers $\tilde{r}(j)$ so that $\tilde{r}(j) \equiv 2 j t \bmod 2 p$ and 
$-p<\tilde{r}(j)<p$. For $1 \leq k \leq p^{\prime}$, there exists a unique $1 \leq j \leq p^{\prime}$ such that $2 k \equiv|\tilde{r}(j)| \bmod 2 p$, and then $i_{k}{ }^{\prime} b=j b$ and $i_{k}{ }^{\prime} d=j d$. We have the following lemma.

Lemma 3. (1) $\sigma_{j c}=\sigma_{j a}=(-1)^{[(2 j-1) t / p]}$, and $\sigma_{j d}=\sigma_{j b}=(-1)^{[2 j t / p]}$.

(2) $\sigma_{j a}=\sigma_{\left(p^{\prime}+1-j\right) b}$ and $\sigma_{i_{k}{ }^{\prime} b}=\sigma_{i_{p^{\prime}+1-k}}$.

Proof. (1) It is clear from the definition.

(2) Since $t$ is odd, $2\left(p^{\prime}+1-j\right) t=(p+1-2 j) t \equiv p t+(1-t) p-(2 j-1) t=p-(2 j-1) t$ $\bmod 2 p$. If $2 j t \equiv \pm 2 k \bmod 2 p$ for $1 \leq k \leq p^{\prime}, 2\left(p^{\prime}+1-k\right)-1=p-2 k \equiv p \mp 2 j t \equiv$ $p \mp 2 j t+(t-1) p \equiv(p \mp 2 j) t \bmod 2 p$. So the claim holds.

Figure 4. $p<2 t$

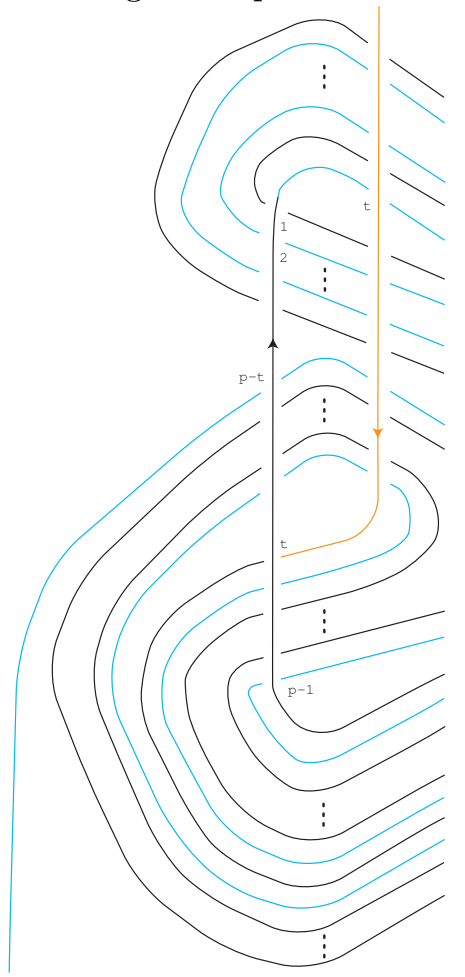

Figure 5. $p>2 t$

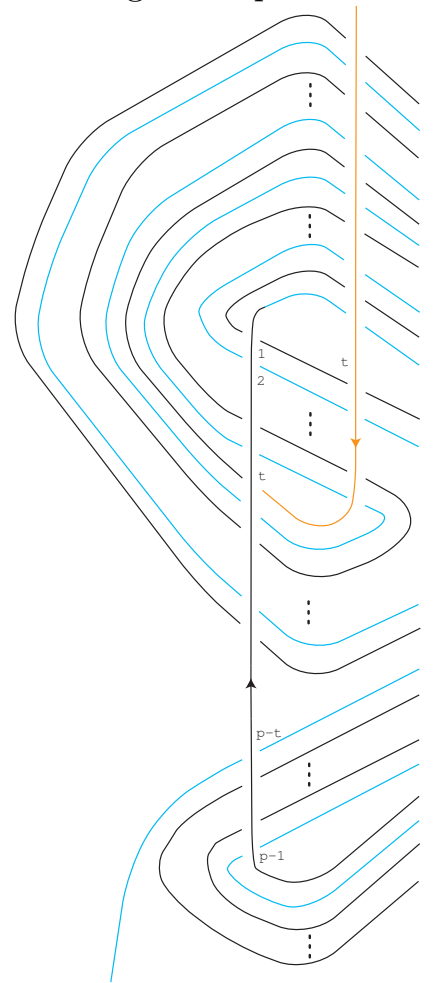

Let us compute $s(j a), s(j b), s(j c), s(j d)$ for $j=1, \cdots, p^{\prime}$. Since the term with $s(j)<0$ does not contribute to the sum in the formula $(2), s(1 a)=-\sum_{j=1}^{p^{\prime}}\left(l_{j b}+l_{j c}\right)$ implies that $l_{j b}, l_{j c}=0$ for $j=1, \cdots, p^{\prime}$ and $s(1 a)=0$. It also follows that $s(j b)=s((j+1) a)=\sum_{k=1}^{j} l_{k a}$ for $1 \leq j \leq p^{\prime}-1$ and $s\left(p^{\prime} b\right)=\sum_{k=1}^{p^{\prime}} l_{k a}$. We also have $s(1 c)=-\sum_{j=1}^{p^{\prime}} l_{j d}$ and so it follows that $l_{j d}=0$ for $1 \leq j \leq p^{\prime}$ and $s(1 c)=0$. Thus only parameters $l_{j a}$ appear in the formula (2). We may denote $l_{j a}$ by simply 


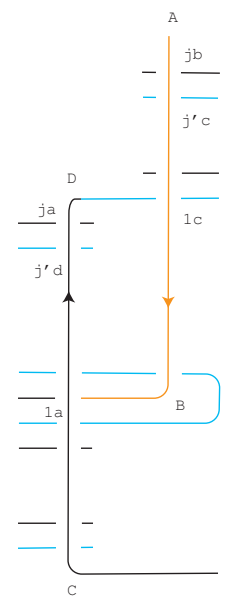

Figure 6.

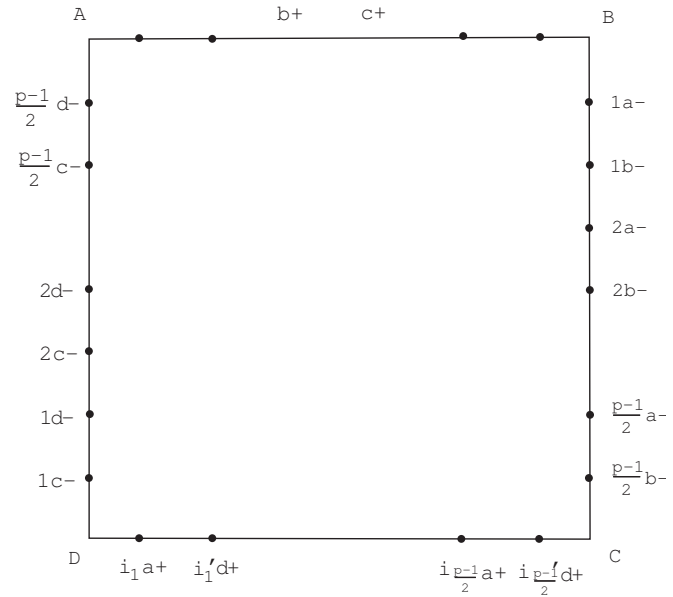

Figure 7.

$l_{j}$ for $1 \leq j \leq p^{\prime}$. Moreover, we compute terms in $C^{0}(\mathbf{l})$. If $j^{\prime} a+<j a+$, then $\pm l_{j^{\prime} a}$ appear in the sum $\sum_{i<j a+} \varepsilon_{i} l_{i}$. So, we have

$$
\sum_{i<j a+} \varepsilon_{i} l_{i}=-\sum_{j^{\prime} a+\geq j a+} l_{j^{\prime}}=-\sum_{r^{\prime}\left(j^{\prime}\right) \leq r^{\prime}(j)} l_{j^{\prime}}=-\sum_{k=1}^{r^{\prime}(j)} l_{i_{k}} .
$$

Similarly, noting that $l_{j d}=0$ and that $j d$ corresponds to $2 j t$ on the segment $C D$, we have

$$
\sum_{i<j d+} \varepsilon_{i} l_{i}=-\sum_{r^{\prime}\left(j^{\prime}\right) \leq r^{\prime}(j)} l_{j^{\prime}}=-\sum_{k=1}^{r^{\prime}(j)} l_{i_{k}}
$$

An easy observation gives

$$
\sum_{i<j b+} \varepsilon_{i} l_{i}=\sum_{i<j c+} \varepsilon_{i} l_{i}=0
$$

So, from $(3),(4),(5)$ and $s(j c)=s(j d)=0$,

$$
\sum_{j} \sigma_{j}\left(\sum_{i<j+} \varepsilon_{i} l_{i}\right)\left(\sum_{k<j-} \varepsilon_{k} l_{k}\right)=\sum_{j=1}^{p^{\prime}} \sigma_{j a}\left(-\sum_{k=1}^{r^{\prime}(j)} l_{i_{k}}\right)(-s(j a)) .
$$

(Recall that $i_{r^{\prime}(j)}=j$ ). Now we compute $q_{j}$. Since $j a+>j a-$ for any $j a$, using 
Lemma 3, we get

$$
\begin{aligned}
q_{j a} & =\frac{1}{2}\left(\sum_{i>j a+} \sigma_{i}-\sum_{i>j a-} \sigma_{i}-\sigma_{j a}\right) \\
& =-\frac{1}{2} \sum_{j a+\geq i \geq j a-} \sigma_{i} \\
& =-\frac{1}{2}\left(\sum_{k=r^{\prime}(j)}^{p^{\prime}}\left(\sigma_{i_{k} a}+\sigma_{i_{k}^{\prime} b}\right)+\sum_{k=j}^{p^{\prime}}\left(\sigma_{k a}+\sigma_{k b}\right)\right) \\
& =-\frac{1}{2}\left(\sum_{k=r^{\prime}(j)}^{p^{\prime}}\left(\sigma_{i_{k} a}+\sigma_{i_{p^{\prime}+1-k} a}\right)+\sum_{k=j}^{p^{\prime}}\left(\sigma_{k a}+\sigma_{\left(p^{\prime}+1-k\right) a}\right)\right) .
\end{aligned}
$$

We will write $\sigma_{j a}=\sigma_{j}$.

We consider the case where $p<2 t$. Let us calculate $\sum_{a} \delta_{a}$. From Figure 4 , a blob of type (i) appears when the sign of the crossing corresponding to $k$ on the segment $C D$ with $1 \leq k \leq p-t-1$ is -1 and when the sign of the crossing corresponding to $k$ on the segment $C D$ with $p-t+1 \leq k \leq t-1$ is +1 . Note that the blobs in the later case appear between the segment $A B$ and $C D$ (see Figrue 8 and 9). A blob of type (ii) appears when the sign of the crossing corresponding to $k$ on the segment $C D$ with $p-t+1 \leq k \leq p-1$ is -1 . Moreover one blob of the type (i) appears before the curve $v_{2}$ reaches the crossing $p^{\prime} d$ and one blob of type (ii) appears when the curve $v_{1}$ meets the point $C$.

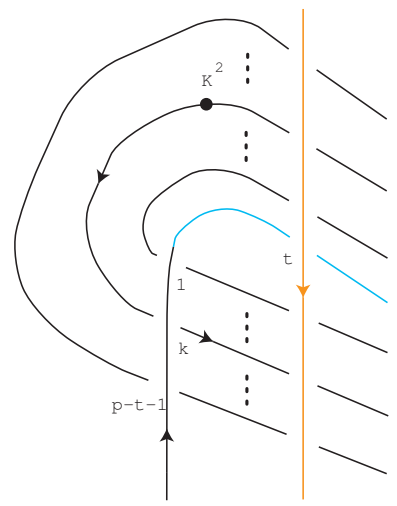

Figure 8.

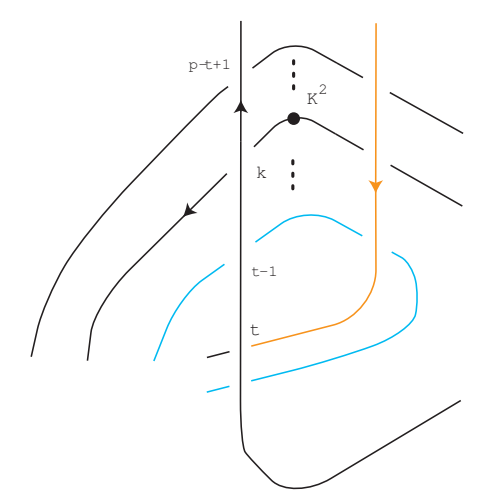

Figure 9 . 
Thus

$$
\begin{aligned}
\sum \delta_{a}= & \sum_{1 \leq k \leq \frac{p-t}{2}} \frac{1-\sigma_{i_{k}}}{2}-\sum_{\frac{p-t}{2}+1 \leq k \leq p^{\prime}} \frac{1-\sigma_{i_{k}}}{2}+\sum_{\frac{p-t}{2}+1 \leq k \leq \frac{t-1}{2}} \frac{1+\sigma_{i_{k}}}{2} \\
& +\sum_{1 \leq k \leq \frac{p-t}{2}-1} \frac{1-\sigma_{i_{p^{\prime}+1-k}}}{2}-\sum_{\frac{p-t}{2}+1 \leq k \leq p^{\prime}} \frac{1-\sigma_{i_{p^{\prime}+1-k}}}{2}+\sum_{\frac{p-t}{2}+1 \leq k \leq \frac{t-1}{2}} \frac{1+\sigma_{i_{p^{\prime}+1-k}}}{2} \\
= & \sum_{1 \leq k \leq \frac{p-t}{2}} \frac{1-\sigma_{i_{k}}}{2}+\sum_{\frac{p-t}{2}+1 \leq k \leq \frac{t-1}{2}} \sigma_{i_{k}}-\sum_{\frac{t+1}{2}+1 \leq k \leq p^{\prime}} \frac{1-\sigma_{i_{k}}}{2} \\
& +\sum_{1 \leq k \leq \frac{p-t}{2}-1} \frac{1-\sigma_{i_{p^{\prime}+1-k}}}{2}+\sum_{\frac{p-t}{2}+1 \leq k \leq \frac{t-1}{2}} \sigma_{i_{p^{\prime}+1-k}}-\sum_{\frac{t+1}{2} \leq k \leq p^{\prime}} \frac{1-\sigma_{i_{p^{\prime}+1-k}}}{2} \\
= & 2 \sum_{\frac{p-t}{2}+1 \leq k \leq \frac{t-1}{2}} \sigma_{i_{k}} .
\end{aligned}
$$

Here we used Lemma 3.

It is easy to see that $\sum \sigma_{j}=4 \sum_{j=1}^{p^{\prime}} \sigma_{j a}$. Therefore, we obtain

$$
\begin{aligned}
d(\mathbf{l})= & -\frac{1}{2} \sum_{j=1}^{p^{\prime}}\left\{\sum_{k=r^{\prime}(j)}^{p^{\prime}}\left(\sigma_{i_{k}}+\sigma_{i_{p^{\prime}+1-k}}\right)+\sum_{k=j}^{p^{\prime}}\left(\sigma_{k}+\sigma_{p^{\prime}+1-k}\right)\right\} l_{j} \\
& -\sum_{\frac{p-t}{2}+1 \leq k \leq \frac{t-1}{2}} \sigma_{i_{k}}-2 \sum_{j=1}^{p^{\prime}} \sigma_{j} \\
= & -\frac{1}{2} \sum_{j=1}^{p^{\prime}}\left\{\sum_{k=r^{\prime}(j)}^{p^{\prime}}\left(\sigma_{i_{k}}+\sigma_{i_{p^{\prime}+1-k}}\right)+\sum_{k=j}^{p^{\prime}}\left(\sigma_{k}+\sigma_{p^{\prime}+1-k}\right)\right\} l_{j}-\sum_{j=1}^{p^{\prime}} \sigma_{j} .
\end{aligned}
$$

To show the second equality above, we need the following lemma.

$$
\begin{aligned}
& \text { Lemma 4. (1) If } p<2 t \text {, then } \sum_{\frac{p-t}{2}+1 \leq k \leq \frac{t-1}{2}} \sigma_{i_{k}}=-\sum_{j=1}^{p^{\prime}} \sigma_{j} \text {. } \\
& \text { (2) If } p>2 t \text {, then } \sum_{\frac{t+1}{2} \leq k \leq \frac{p-t}{2}} \sigma_{i_{k}}=\sum_{j=1}^{p^{\prime}} \sigma_{j} .
\end{aligned}
$$

Proof. In the case where $p<2 t$, we focus the signs of the crossings labeled by even on the segment $w_{1}$. Note that the sum of their signs equals $\sum_{j=1}^{p^{\prime}} \sigma_{j}$. On the other hand, from Figure 4, it follows that the sum of the signs of the crossings labeled even $j$ so that $2 t-p+1 \leq j \leq p-1$ equals 0 and that the sum of the sign of the crossing labeled even $j$ so that $2 \leq j \leq 2 t-p-1$ equals $-\sum_{\frac{p-t}{2}+1 \leq k \leq \frac{t-1}{2}} \sigma_{i_{k}}$. So 
(1) holds. Similarly, in the case where $p>2 t$, we examine the sum of the sign of the crossing labeled even on the segment $w_{1}$ in Figure 5 . Note that the sum of their signs equals $\sum_{j=1}^{p^{\prime}} \sigma_{j}$. From the diagram, it follows that the sum of the sign of the crossing labeled even $j$ so that $2 \leq j \leq 2 t$ equals 0 and that the sum of the sign of the crossing labeled even $j$ so that $2 t+2 \leq j \leq p-1$ equals $\sum_{\frac{t+1}{2} \leq k \leq \frac{p-t}{2}} \sigma_{i_{k}}$. So we get the equality (2).

Next, we calculate the term $\sum_{a} \delta_{a} \sum_{k<i_{a}} \varepsilon_{k} l_{k}$, which comes from the crossing of type (2) on $C D$ and blobs of both types on the curve $v_{1}$. All blobs of type (i) give

$$
-\sum_{1 \leq k \leq \frac{p-t}{2}} \frac{1-\sigma_{i_{k}}}{2} \sum_{1 \leq s \leq i_{k}-1} l_{s}-\sum_{\frac{p-t}{2}+1 \leq k \leq \frac{t-1}{2}} \frac{1+\sigma_{i_{k}}}{2} \sum_{1 \leq s \leq i_{k}-1} l_{s},
$$

and all blobs of type (ii) except the special blob of type (ii) near $C$ give

$$
\sum_{\frac{p-t}{2}+1 \leq k \leq p^{\prime}} \frac{1-\sigma_{i_{k}}}{2} \sum_{1 \leq s \leq i_{k}-1} l_{s}
$$

and all crossings of type (2) give

$$
\sum_{\frac{p-t}{2}+1 \leq k \leq p^{\prime}} \frac{1+\sigma_{i_{k}}}{2}\left(-l_{i_{k}}\right),
$$

and the special blob gives $\sum_{j=1}^{p^{\prime}} l_{j}$.

Moreover, we compute the term $\sum_{k<i} \sigma_{i} \varepsilon_{i} l_{i} \varepsilon_{k} l_{k}$. When $i<1 a-, i>i_{1} a+$, $i=j b-$, we have $\sigma_{i} \varepsilon_{i} l_{i}=0$. If $i=1 a-$, then $\sum_{k<i} \sigma_{i} \varepsilon_{i} l_{i} \varepsilon_{k} l_{k}=0$. If $i=j a-$ for $2 \leq j \leq p^{\prime}$

$$
\sigma_{i} \varepsilon_{i} l_{i} \sum_{k<i} \varepsilon_{k} l_{k}=\sigma_{j}(-1) l_{j}\left(-\sum_{k=1}^{j-1} l_{k}\right)
$$

and if $i=i_{k^{\prime}} a+$ for $1 \leq k^{\prime} \leq p^{\prime}$,

$$
\sigma_{i} \varepsilon_{i} l_{i} \sum_{k<i} \varepsilon_{k} l_{k}=\sigma_{i_{k^{\prime}}} l_{i_{k^{\prime}}}\left(-\sum_{k=1}^{k^{\prime}} l_{i_{k}}\right) .
$$

We remark that $i_{k^{\prime}+1} a \pm, \cdots, i_{p^{\prime}} a \pm$ appear in the sum in the left hand side of (8). We can see that the term $\sigma_{i_{k}} l_{i_{k}}\left(-l_{i_{m}}\right)$ with $k<m$ and $j^{\prime}=i_{k}>i_{m}=j$ in (8) is 
cancelled with the term $\sigma_{j^{\prime}}(-1) l_{j^{\prime}}\left(-l_{j}\right)$ in (7). Thus, we get

$$
\begin{aligned}
\sum_{\substack{i \\
k<i}} \sigma_{i} \varepsilon_{i} l_{i} \varepsilon_{k} l_{k} & =-\sum_{j=1}^{p^{\prime}} \sigma_{j} l_{j}^{2}+\sum_{\substack{j>j^{\prime} \\
j a+>j^{\prime} a+}}\left(\sigma_{j a}-\sigma_{j^{\prime} a}\right) l_{j a} l_{j^{\prime} a} \\
& =-\sum_{j=1}^{p^{\prime}} \sigma_{j} l_{j}^{2}+\sum_{1 \leq k \leq p^{\prime}-1} \sum_{k+1 \leq k^{\prime} \leq p^{\prime}} \frac{1+\operatorname{sign}\left(i_{k}-i_{k^{\prime}}\right)}{2}\left(\sigma_{i_{k}}-\sigma_{i_{k^{\prime}}}\right) l_{i_{k}} l_{i_{k^{\prime}}} .
\end{aligned}
$$

Finally, we get

$$
\begin{aligned}
C^{0}(\mathbf{l})= & -d(\mathbf{l})+\sum_{1 \leq k \leq \frac{p-t}{2}} \frac{1-\sigma_{i_{k}}}{2} \sum_{1 \leq s \leq i_{k}-1} l_{s}+\sum_{\frac{p-t}{2}+1 \leq k \leq \frac{t-1}{2}} \frac{1+\sigma_{i_{k}}}{2} \sum_{1 \leq s \leq i_{k}-1} l_{s} \\
& -\sum_{\frac{p-t}{2}+1 \leq k \leq p^{\prime}} \frac{1-\sigma_{i_{k}}}{2} \sum_{1 \leq s \leq i_{k}-1} l_{s}+\sum_{\frac{p-t}{2}+1 \leq k \leq p^{\prime}} \frac{1+\sigma_{i_{k}}}{2} l_{i_{k}}-\sum_{j=1}^{p^{\prime}} l_{j}-\frac{1}{2} \sum_{j=1}^{p^{\prime}} \sigma_{j} l_{j} \\
& -\frac{1}{2}\left\{-\sum_{j=1}^{p^{\prime}} \sigma_{j} l_{j}^{2}+\sum_{1 \leq k \leq p^{\prime}-1} \sum_{k+1 \leq k^{\prime} \leq p^{\prime}} \frac{1+\operatorname{sign}\left(i_{k}-i_{k^{\prime}}\right)}{2}\left(\sigma_{i_{k}}-\sigma_{i_{k^{\prime}}}\right) l_{i_{k}} l_{i_{k^{\prime}}}\right\} \\
& +\sum_{1 \leq k \leq p^{\prime}} \sigma_{j}\left(-\sum_{k=1}^{j-1} l_{i_{k}}\right)\left(-\sum_{k=1}^{r^{\prime}(j)} l_{i_{k}}\right) \\
= & -d(\mathbf{l})+\sum_{1 \leq k \leq \frac{p-t}{2}} \frac{1-\sigma_{i_{k}}}{2} \sum_{1 \leq s \leq i_{k}-1} l_{s}+\sum_{\frac{p-t}{2}+1 \leq k \leq \frac{t-1}{2}} \frac{1+\sigma_{i_{k}}}{2} \sum_{1 \leq s \leq i_{k}-1} l_{s} \\
& +\sum_{\frac{p-t}{2}+1 \leq k \leq p^{\prime}} \frac{1+\sigma_{i_{k}}}{2} l_{i_{k}}-\sum_{\frac{p-t}{2}+1 \leq k \leq p^{\prime}} \frac{1-\sigma_{i_{k}}}{2} l_{s}-\sum_{j=1}^{p^{\prime}} l_{j}-\frac{1}{2} \sum_{j=1}^{p^{\prime}} \sigma_{j} l_{j} \\
& +\frac{1}{2} \sum_{j=1}^{p^{\prime}} \sigma_{j} l_{j}^{2}-\frac{1}{2}\left\{\sum_{1 \leq k \leq p^{\prime}-1} \sum_{k+1 \leq k^{\prime} \leq p^{\prime}} \frac{1+\operatorname{sign}\left(i_{k}-i_{k^{\prime}}\right)}{2}\left(\sigma_{i_{k}}-\sigma_{i_{k^{\prime}}}\right) l_{i_{k}} l_{i_{k^{\prime}}}\right\} \\
& +\sum_{1 \leq k \leq p^{\prime}} \sigma_{j}\left(\sum_{k=1}^{j-1} l_{i_{k}}\right)\left(\sum_{k=1}^{r^{\prime}(j)} l_{i_{k}}\right) .
\end{aligned}
$$

Similarly, we compute $d(\mathbf{l})$ and $C^{0}(\mathbf{l})$ in the case where $p>2 t$. From Figure 5 , one can see that a blob of type (i) appears when the sign of the crossing corresponding to $k$ on the segment $C D$ with $1 \leq k \leq p-t-1$ is -1 . A blob of type (ii) appears when the sign of the crossing corresponding to $k$ on the segment $C D$ with $p-t+1 \leq k \leq p-1$ is -1 and when the sign of the crossing corresponding to $k$ on the segment $C D$ with $t+1 \leq k \leq p-t-1$ is +1 . Moreover one blob of the 
type (ii) appears before the curve $v_{1}$ arrives at the crossing $1 a$ and one blob of the same type appears when the curve $v_{1}$ meets the point $C$. Therefore, by Lemma 3 , it follows that

$$
\begin{aligned}
\sum \delta_{a}= & \sum_{1 \leq k \leq \frac{p-t}{2}} \frac{1-\sigma_{i_{k}}}{2}-\sum_{\frac{p-t}{2}+1 \leq k \leq p^{\prime}} \frac{1-\sigma_{i_{k}}}{2}-\sum_{\frac{t+1}{2}+1 \leq k \leq \frac{p-t}{2}} \frac{1+\sigma_{i_{k}}}{2} \\
& +\sum_{1 \leq k \leq \frac{p-t}{2}-1} \frac{1-\sigma_{i_{p^{\prime}+1-k}}}{2}-\sum_{\frac{p-t}{2}+1 \leq k \leq p^{\prime}} \frac{1-\sigma_{i_{p^{\prime}+1-k}}}{2} \\
& -\sum_{\frac{t+1}{2} \leq k \leq \frac{p-t}{2}-1} \frac{1+\sigma_{i_{p^{\prime}+1-k}}}{2}-2 \\
= & \sum_{1 \leq k \leq \frac{t+1}{2}} \frac{1-\sigma_{i_{k}}}{2}-\sum_{\frac{t+1}{2}+1 \leq k \leq \frac{p-t}{2}} \sigma_{i_{k}}-\sum_{\frac{p-t}{2}+1 \leq k \leq p^{\prime}} \frac{1-\sigma_{i_{k}}}{2} \\
& +\sum_{1 \leq k \leq \frac{t-1}{2}} \frac{1-\sigma_{i_{p^{\prime}+1-k}}}{2}-\sum_{\frac{t+1}{2} \leq k \leq \frac{p-t}{2}-1} \sigma_{i_{p^{\prime}+1-k}}-\sum_{\frac{p-t}{2}+1 \leq k \leq p^{\prime}} \frac{1-\sigma_{i_{p^{\prime}+1-k}}}{2}-2 \\
= & -2 \sum_{\frac{t+1}{2} \leq k \leq \frac{p-t}{2}} \sigma_{i_{k}} .
\end{aligned}
$$

Here we used $\sigma_{\frac{i_{t+1}}{2}}=1$ in the last equality. Accordingly, from Lemma 4, we obtain

$$
\begin{aligned}
d(\mathbf{l})= & -\frac{1}{2} \sum_{j=1}^{p^{\prime}}\left\{\sum_{k=r^{\prime}(j)}^{p^{\prime}}\left(\sigma_{i_{k}}+\sigma_{i_{p^{\prime}-k}}\right)+\sum_{k=j}^{p^{\prime}}\left(\sigma_{k}+\sigma_{p^{\prime}+1-k}\right)\right\} l_{j} \\
& +\sum_{\frac{t+1}{2}+1 \leq k \leq \frac{p-t}{2}} \sigma_{i_{k}}-2 \sum_{j=1}^{p^{\prime}} \sigma_{j} \\
= & -\frac{1}{2} \sum_{j=1}^{p^{\prime}}\left\{\sum_{k=r^{\prime}(j)}^{p^{\prime}}\left(\sigma_{i_{k}}+\sigma_{i_{p^{\prime}+1-k}}\right)+\sum_{k=j}^{p^{\prime}}\left(\sigma_{k}+\sigma_{p^{\prime}+1-k}\right)\right\} l_{j}-\sum_{j=1}^{p^{\prime}} \sigma_{j} .
\end{aligned}
$$

Next, we calculate the term $\sum_{a} \delta_{a} \sum_{k \leq i_{a}} \varepsilon_{k} l_{k}$. Non-trivial terms correspond to the crossing of type (2) and blobs of both type on the curve $v_{1}$. All blobs of type (i) give

$$
-\sum_{1 \leq k \leq \frac{p-t}{2}} \frac{1-\sigma_{i_{k}}}{2} \sum_{1 \leq s \leq i_{k}-1} l_{s}
$$

and all blobs of type (ii) except the special blob near $C$ give

$$
\sum_{\frac{p-t}{2}+1 \leq k \leq p^{\prime}} \frac{1-\sigma_{i_{k}}}{2} \sum_{1 \leq s \leq i_{k}-1} l_{s}+\sum_{\frac{t+1}{2}+1 \leq k \leq \frac{p-t}{2}} \frac{1+\sigma_{i_{k}}}{2} \sum_{1 \leq s \leq i_{k}-1} l_{s}
$$


and all crossings of type (2) give

$$
\sum_{\frac{p-t}{2}+1 \leq k \leq p^{\prime}} \frac{1+\sigma_{i_{k}}}{2}\left(-l_{i_{k}}\right),
$$

and the special blob gives $\sum_{j=1}^{p^{\prime}} l_{j}$.

From Figure 7, we can see

$$
\begin{aligned}
& \sum_{\substack{i \\
k<i_{a} \\
k}} \sigma_{i} \varepsilon_{i} l_{i} \varepsilon_{k} l_{k} \\
= & -\sum_{j=1}^{p^{\prime}} \sigma_{j} l_{j}^{2}+\sum_{\substack{j>j^{\prime} \\
j a+>j^{\prime} a+}}\left(\sigma_{j a}-\sigma_{j^{\prime} a}\right) l_{j a} l_{j^{\prime} a} \\
= & -\sum_{j=1}^{p^{\prime}} \sigma_{j} l_{j}^{2}+\sum_{1 \leq k \leq p^{\prime}-1} \sum_{k+1 \leq k^{\prime} \leq p^{\prime}} \frac{1+\operatorname{sign}\left(i_{k}-i_{k^{\prime}}\right)}{2}\left(\sigma_{i_{k}}-\sigma_{i_{k^{\prime}}}\right) l_{i_{k}} l_{i_{k^{\prime}}},
\end{aligned}
$$

and so it is obtained that

$$
\begin{aligned}
C^{0}(\mathbf{l})= & -d(\mathbf{l}) \\
& +\sum_{1 \leq k \leq \frac{p-t}{2}} \frac{1-\sigma_{i_{k}}}{2} \sum_{1 \leq s \leq i_{k}-1} l_{s}-\sum_{\frac{p-t}{2}+1 \leq k \leq p^{\prime}} \frac{1-\sigma_{i_{k}}}{2} \sum_{1 \leq s \leq i_{k}-1} l_{s} \\
& -\sum_{\frac{t+1}{2}+1 \leq k \leq \frac{p-t}{2}} \frac{1+\sigma_{i_{k}}}{2} \sum_{1 \leq s \leq i_{k}-1} l_{s}-\sum_{\frac{p-t}{2}+1 \leq k \leq p^{\prime}} \frac{1+\sigma_{i_{k}}}{2}\left(-l_{i_{k}}\right) \\
& -\sum_{j=1}^{p^{\prime}} l_{j}-\frac{1}{2} \sum_{j=1}^{p^{\prime}} \sigma_{j} l_{j} \\
& -\frac{1}{2}\left\{-\sum_{j=1}^{p^{\prime}} \sigma_{j} l_{j}^{2}+\sum_{1 \leq k \leq p^{\prime}} \sum_{k+1 \leq k^{\prime} \leq p^{\prime}} \frac{1+\operatorname{sign}\left(i_{k}-i_{k^{\prime}}\right)}{2}\left(\sigma_{i_{k}}-\sigma_{i_{k^{\prime}}}\right) l_{i_{k}} l_{i_{k^{\prime}}}\right\} \\
& +\sum_{1 \leq k \leq p^{\prime}} \sigma_{j}\left(-\sum_{k=1}^{j-1} l_{k}\right)\left(-\sum_{k=1}^{r^{\prime}(j)} l_{i_{k}}\right) \\
= & -d(\mathbf{l})+\sum_{1 \leq k \leq \frac{p-t}{2}} \frac{1-\sigma_{i_{k}}}{2} \sum_{1 \leq s \leq i_{k}-1} l_{s}+\sum_{\frac{p-t}{2}+1 \leq k \leq p^{\prime}} \frac{1+\sigma_{i_{k}}}{2} l_{i_{k}} \\
& -\sum_{\frac{p-t}{2}+1 \leq k \leq p^{\prime}} \frac{1-\sigma_{i_{k}}}{2} \sum_{1 \leq s \leq i_{k}-1} l_{s}-\sum_{\frac{t+1}{2}+1 \leq k \leq \frac{p-t}{2}} \frac{1+\sigma_{i_{k}}}{2} \sum_{1 \leq s \leq i_{k}-1} l_{s}
\end{aligned}
$$




$$
\begin{aligned}
& -\sum_{j=1}^{p^{\prime}} l_{j}-\frac{1}{2} \sum_{j=1}^{p^{\prime}} \sigma_{j} l_{j}+\frac{1}{2} \sum_{j=1}^{p^{\prime}} \sigma_{j} l_{j}^{2} \\
& -\frac{1}{2}\left\{\sum_{1 \leq k \leq p^{\prime}} \sum_{k+1 \leq k^{\prime} \leq p^{\prime}} \frac{1+\operatorname{sign}\left(i_{k}-i_{k^{\prime}}\right)}{2}\left(\sigma_{i_{k}}-\sigma_{i_{k^{\prime}}}\right) l_{i_{k}} l_{i_{k^{\prime}}}\right\} \\
& +\sum_{1 \leq k \leq p^{\prime}} \sigma_{j}\left(\sum_{k=1}^{j-1} l_{k}\right)\left(\sum_{k=1}^{r^{\prime}(j)} l_{i_{k}}\right) .
\end{aligned}
$$

We compute the remaining term in the formula (2). Since $\sigma_{1}=1, s(1 a)=0$, and $s(j a)=\sum_{k=1}^{j-1} l_{k}\left(2 \leq j \leq p^{\prime}\right)$

$$
\begin{aligned}
& \prod_{j=1}^{c}\left\{\begin{array}{c}
s(j)+l_{j} \\
l_{j}
\end{array}\right\}_{\sigma_{j}}\left(q^{\sigma_{j}(n-s(j)-1)}-1\right) \cdots\left(q^{\sigma_{j}\left(n-s(j)-l_{j}\right)}-1\right) \\
= & \prod_{j=1}^{p^{\prime}}\left\{\begin{array}{c}
\sum_{k=1}^{j} l_{k} \\
l_{j}
\end{array}\right\}_{\sigma_{j}}\left(q^{\sigma_{j}\left(n-\sum_{k=1}^{j-1} l_{k}-1\right)}-1\right) \cdots\left(q^{\sigma_{j}\left(n-\sum_{k=1}^{j-1} l_{k}-l_{j}\right)}-1\right) .
\end{aligned}
$$

Putting $m_{j}:=\sum_{k=1}^{j} l_{k}$ and $m_{0}=0$, the right hand side is changed to

$$
\prod_{j=1}^{p^{\prime}}\left\{\begin{array}{c}
m_{j} \\
m_{j}-m_{j-1}
\end{array}\right\}_{\sigma_{j}}\left(q^{\sigma_{j}\left(n-m_{j-1}-1\right)}-1\right) \cdots\left(q^{\sigma_{j}\left(n-m_{j}\right)}-1\right) .
$$

Noting that

$$
\begin{aligned}
& \left(q^{\sigma_{j}\left(n-m_{j-1}-1\right)}-1\right) \cdots\left(q^{\sigma_{j}\left(n-m_{j}\right)}-1\right)=\frac{\left(q^{\sigma_{j}}\right)_{n-m_{j-1}-1}}{\left(q^{\sigma_{j}}\right)_{n-m_{j}-1}}, \\
& \left\{\begin{array}{c}
m_{j} \\
m_{j}-m_{j-1}
\end{array}\right\}_{\sigma_{j}}=\frac{\left(q^{\sigma_{j}}\right)_{m_{j}}}{\left(q^{\sigma_{j}}\right)_{m_{j}-m_{j-1}}\left(q^{\sigma_{j}}\right)_{m_{j-1}}}, \\
& \frac{\left(q^{\sigma}\right)_{m}}{\left(q^{\sigma^{\prime}}\right)_{m}}=(-1)^{\frac{\sigma-\sigma^{\prime}}{2} m} q^{\frac{\sigma-\sigma^{\prime}}{2} \cdot \frac{1}{2} m(m+1)},
\end{aligned}
$$

(9) can be exchanged to

$$
\frac{\left(q^{\sigma_{p^{\prime}}}\right)_{n-1}\left(q^{\sigma_{p^{\prime}}}\right)_{m_{p^{\prime}}}}{\left(q^{\sigma_{p^{\prime}}}\right)_{n-m_{p^{\prime}}-1}}\left(\prod_{j=1}^{p^{\prime}}\left(q^{\sigma_{j}}\right)_{m_{j}-m_{j-1}}\right)^{-1}
$$




$$
\begin{aligned}
& \times \prod_{j=2}^{p^{\prime}-1}(-1)^{\frac{\sigma_{j}-\sigma_{j+1}}{2} m_{j}} q^{\frac{\sigma_{j}-\sigma_{j+1}}{2} \frac{1}{2} m_{j}\left(m_{j}+1\right)} \\
& \times \prod_{j=2}^{p^{\prime}-1}(-1)^{\frac{\sigma_{j+1}-\sigma_{j}}{2}\left(n-m_{j}-1\right)} q^{\frac{\sigma_{j+1}-\sigma_{j}}{2}} \frac{1}{2}\left(n-m_{j}\right)\left(n-m_{j}-1\right) \\
= & \frac{\left(q^{\sigma_{p^{\prime}}}\right)_{n-1}\left(q^{\sigma_{p^{\prime}}}\right)_{m_{p^{\prime}}}}{\left(q^{\sigma_{p^{\prime}}}\right)_{n-m_{p^{\prime}}-1}}\left(\prod_{j=1}^{p^{\prime}}\left(q^{\sigma_{j}}\right)_{m_{j}-m_{j-1}}\right)^{-1} q^{-n \sum_{j=1}^{p^{\prime}-1} \frac{\sigma_{j+1}-\sigma_{j}}{2} m_{j}} .
\end{aligned}
$$

Here we used $\sigma_{2}=\sigma_{p^{\prime}}=-1$ if $p<2 t$ and $\sigma_{2}=\sigma_{p^{\prime}}=1$ if $p>2 t$. Recalling $m_{j}-m_{j-1}=l_{j}$, it is changed to

$$
\begin{aligned}
& \frac{\left(q^{\sigma_{p^{\prime}}}\right)_{n-1}\left(q^{\sigma_{p^{\prime}}}\right)_{m_{p^{\prime}}}}{\left(q^{\sigma_{p^{\prime}}}\right)_{n-m_{p^{\prime}}-1}}(-1)^{\sum_{j=1}^{p^{\prime}} l_{j}} q^{-\sum_{j=1}^{p^{\prime}} \frac{1}{2} \sigma_{j} l_{j}\left(l_{j}+1\right)} \\
& \times\left(\prod_{j=1}^{p^{\prime}}\left(q^{-\sigma_{j}}\right)_{m_{j}-m_{j-1}}\right)^{-1} q^{-n \sum_{j=1}^{p^{\prime}-1} \frac{\sigma_{j+1}-\sigma_{j}}{2} m_{j}} .
\end{aligned}
$$

Accordingly, we obtain

$$
\begin{aligned}
& d(\mathbf{l})-\sum_{j=1}^{p^{\prime}-1} \frac{\sigma_{j+1}-\sigma_{j}}{2} m_{j} \\
= & -\frac{1}{2} \sum_{j=1}^{p^{\prime}}\left\{\sum_{k=r^{\prime}(j)}^{p^{\prime}}\left(\sigma_{i_{k}}+\sigma_{i_{p^{\prime}+1-k}}\right)+\sum_{k=j}^{p^{\prime}}\left(\sigma_{k}+\sigma_{p^{\prime}+1-k}\right)\right\}\left(m_{j}-m_{j-1}\right) \\
& -\sum_{j=1}^{p^{\prime}} \sigma_{j}-\sum_{j=1}^{p^{\prime}-1} \frac{\sigma_{j+1}-\sigma_{j}}{2} m_{j} \\
= & -\frac{1}{2} \sum_{j=1}^{p^{\prime}} \sum_{k=r^{\prime}(j)}^{p^{\prime}}\left(\sigma_{i_{k}}+\sigma_{i_{p^{\prime}+1-k}}\right)\left(m_{j}-m_{j-1}\right)-\frac{1}{2} \sum_{j=1}^{p^{\prime}} \sum_{k=j}^{p^{\prime}}\left(\sigma_{k}+\sigma_{p^{\prime}+1-k}\right)\left(m_{j}-m_{j-1}\right) \\
& -\sum_{j=1}^{p^{\prime}} \sigma_{j}-\sum_{j=1}^{p^{\prime}-1} \frac{\sigma_{j+1}-\sigma_{j}}{2} m_{j} \\
= & -\frac{1}{2} \sum_{j=1}^{p^{\prime}} \sum_{k=r^{\prime}(j)}^{p^{\prime}}\left(\sigma_{i_{k}}+\sigma_{i_{p^{\prime}+1-k}}\right)\left(m_{j}-m_{j-1}\right)-\frac{1}{2} \sum_{j=1}^{p^{\prime}}\left(\sum_{k=j}^{p^{\prime}}\left(\sigma_{k}+\sigma_{p^{\prime}+1-k}\right) m_{j}\right. \\
& \left.-\sum_{k=j+1}^{p^{\prime}}\left(\sigma_{k}+\sigma_{p^{\prime}+1-k}\right) m_{j}\right)-\sum_{j=1}^{p^{\prime}-1} \sigma_{j}-\sum_{j=1}^{\sigma_{j+1}-\sigma_{j}} m_{j}
\end{aligned}
$$




$$
\begin{aligned}
= & -\frac{1}{2} \sum_{j=1}^{p^{\prime}} \sum_{k=r^{\prime}(j)}^{p^{\prime}}\left(\sigma_{i_{k}}+\sigma_{i_{p^{\prime}+1-k}}\right)\left(m_{j}-m_{j-1}\right)-\frac{1}{2} \sum_{j=1}^{p^{\prime}}\left(\sigma_{j}+\sigma_{p^{\prime}+1-j}\right) m_{j} \\
& -\sum_{j=1}^{p^{\prime}} \sigma_{j}-\frac{1}{2} \sum_{j=1}^{p^{\prime}-1}\left(\sigma_{j+1}-\sigma_{j}\right) m_{j} \\
= & -\frac{1}{2} \sum_{j=1}^{p^{\prime}} \sum_{k=r^{\prime}(j)}^{p^{\prime}}\left(\sigma_{i_{k}}+\sigma_{i_{p^{\prime}+1-k}}\right)\left(m_{j}-m_{j-1}\right) \\
& -\frac{1}{2} \sum_{j=1}^{p^{\prime}-1}\left(\sigma_{j+1}+\sigma_{p^{\prime}+1-j}\right) m_{j}-\frac{1}{2}\left(\sigma_{p^{\prime}}+\sigma_{1}\right) m_{p^{\prime}}-\sum_{j=1}^{p^{\prime}} \sigma_{j} \\
= & a(\mathbf{m}),
\end{aligned}
$$

and for the case where $p<2 t$,

$$
\begin{aligned}
& C^{0}(\mathbf{l})-\sum_{j=1}^{p^{\prime}} \frac{1}{2} \sigma_{j} l_{j}\left(l_{j}+1\right) \\
= & -a(\mathbf{m})-\frac{1}{2} \sum_{j=1}^{p^{\prime}-1}\left(\sigma_{j+1}-\sigma_{j}\right) m_{j} \\
& +\sum_{1 \leq k \leq \frac{p-t}{2}} \frac{1-\sigma_{i_{k}}}{2} m_{i_{k}-1}+\sum_{\frac{p-t}{2}+1 \leq k \leq \frac{t-1}{2}} \frac{1+\sigma_{i_{k}}}{2} m_{i_{k}-1} \\
& +\sum_{\frac{p-t}{2}+1 \leq k \leq p^{\prime}} \frac{1+\sigma_{i_{k}}}{2} m_{i_{k}}-\sum_{\frac{p-t}{2}+1 \leq k \leq p^{\prime}} m_{i_{k}-1}-m_{p^{\prime}}-\sum_{j=1}^{p^{\prime}} \sigma_{j}\left(m_{j}-m_{j-1}\right) \\
& -\frac{1}{2}\left\{\sum_{1 \leq k \leq p^{\prime}} \sum_{k+1 \leq k^{\prime} \leq p^{\prime}} \frac{1+\operatorname{sign}\left(i_{k}-i_{k^{\prime}}\right)}{2}\left(\sigma_{i_{k}}-\sigma_{i_{k^{\prime}}}\right)\left(m_{i_{k}}-m_{i_{k}-1}\right)\left(m_{i_{k^{\prime}}}-m_{i_{k^{\prime}}-1}\right)\right\} \\
& +\sum_{1 \leq k \leq p^{\prime}} \sigma_{j} m_{j-1}\left(\sum_{k=1}^{r^{\prime}(j)}\left(m_{i_{k}}-m_{i_{k}-1}\right)\right) \\
= & -a(\mathbf{m})-\left(1+\sigma_{p^{\prime}}\right) m_{p^{\prime}}+\frac{1}{2} \sum_{j=1}^{p^{\prime}-1}\left(\sigma_{j+1}-\sigma_{j}\right) m_{j} \\
& +\sum_{1 \leq k \leq \frac{p-t}{2}} \frac{1-\sigma_{i_{k}}}{2} m_{i_{k}-1}+\sum_{\frac{p-t}{2}+1 \leq k \leq \frac{t-1}{2}} \frac{1+\sigma_{i_{k}}}{2} m_{i_{k}-1} \\
& +\sum_{\frac{p-t}{2}+1 \leq k \leq p^{\prime}} \frac{1+\sigma_{i_{k}}}{2} m_{i_{k}}-\sum_{\frac{p-t}{2}+1 \leq k \leq p^{\prime}} m_{i_{k}-1}
\end{aligned}
$$




$$
\begin{aligned}
& \quad-\frac{1}{2}\left\{\sum_{1 \leq k \leq p^{\prime}-1} \sum_{k+1 \leq k^{\prime} \leq p^{\prime}} \frac{1+\operatorname{sign}\left(i_{k}-i_{k^{\prime}}\right)}{2}\left(\sigma_{i_{k}}-\sigma_{i_{k^{\prime}}}\right)\left(m_{i_{k}}-m_{i_{k}-1}\right)\left(m_{i_{k^{\prime}}}-m_{i_{k^{\prime}}-1}\right)\right\} \\
& +\sum_{1 \leq k \leq p^{\prime}} \sigma_{j} m_{j-1} \sum_{k=1}^{r^{\prime}(j)}\left(m_{i_{k}}-m_{i_{k}-1}\right) \\
& =b_{1}(\mathbf{m})+b_{2}(\mathbf{m}),
\end{aligned}
$$

and for the case where $p>2 t$,

$$
\begin{aligned}
& C^{0}(\mathbf{l})-\sum_{j=1}^{p^{\prime}} \frac{1}{2} \sigma_{j} l_{j}\left(l_{j}+1\right) \\
& =-a(\mathbf{m})-\frac{1}{2} \sum_{j=1}^{p^{\prime}-1}\left(\sigma_{j+1}-\sigma_{j}\right) m_{j} \\
& +\sum_{1 \leq k \leq \frac{p-t}{2}} \frac{1-\sigma_{i_{k}}}{2} m_{i_{k}-1}+\sum_{\frac{p-t}{2}+1 \leq k \leq \frac{p-1}{2}} \frac{1+\sigma_{i_{k}}}{2} m_{i_{k}}-\sum_{\frac{p-t}{2}+1 \leq k \leq \frac{p-1}{2}} m_{i_{k}-1} \\
& -\sum_{\frac{t+1}{2}+1 \leq k \leq \frac{p-t}{2}} \frac{1+\sigma_{i_{k}}}{2} m_{i_{k}-1}-m_{p^{\prime}}-\sum_{j=1}^{p^{\prime}} \sigma_{j}\left(m_{j}-m_{j-1}\right) \\
& -\frac{1}{2}\left\{\sum_{1 \leq k \leq p^{\prime}} \sum_{k+1 \leq k^{\prime} \leq p^{\prime}} \frac{1+\operatorname{sign}\left(i_{k}-i_{k^{\prime}}\right)}{2}\left(\sigma_{i_{k}}-\sigma_{i_{k^{\prime}}}\right)\left(m_{i_{k}}-m_{i_{k}-1}\right)\left(m_{i_{k^{\prime}}}-m_{i_{k^{\prime}}-1}\right)\right\} \\
& +\sum_{1 \leq k \leq p^{\prime}} \sigma_{j} m_{j-1}\left(\sum_{k=1}^{r^{\prime}(j)}\left(m_{i_{k}}-m_{i_{k}-1}\right)\right) \\
& =-a(\mathbf{m})-\left(1+\sigma_{p^{\prime}}\right) m_{p^{\prime}}+\frac{1}{2} \sum_{j=1}^{p^{\prime}-1}\left(\sigma_{j+1}-\sigma_{j}\right) m_{j} \\
& +\sum_{1 \leq k \leq \frac{p-t}{2}} \frac{1-\sigma_{i_{k}}}{2} m_{i_{k}-1}+\sum_{\frac{p-t}{2}+1 \leq k \leq p^{\prime}} \frac{1+\sigma_{i_{k}}}{2} m_{i_{k}}-\sum_{\frac{p-t}{2}+1 \leq k \leq p^{\prime}} m_{i_{k}-1} \\
& -\sum_{\frac{t+1}{2}+1 \leq k \leq \frac{p-t}{2}} \frac{1+\sigma_{i_{k}}}{2} m_{i_{k}-1} \\
& -\frac{1}{2}\left\{\sum_{1 \leq k \leq p^{\prime}-1} \sum_{k+1 \leq k^{\prime} \leq p^{\prime}} \frac{1+\operatorname{sign}\left(i_{k}-i_{k^{\prime}}\right)}{2}\left(\sigma_{i_{k}}-\sigma_{i_{k^{\prime}}}\right)\left(m_{i_{k}}-m_{i_{k}-1}\right)\left(m_{i_{k^{\prime}}}-m_{i_{k^{\prime}}-1}\right)\right\} \\
& +\sum_{1 \leq k \leq p^{\prime}} \sigma_{j} m_{j-1}\left(\sum_{k=1}^{r^{\prime}(j)}\left(m_{i_{k}}-m_{i_{k}-1}\right)\right)=b_{1}(\mathbf{m})+b_{2}(\mathbf{m}) \text {. }
\end{aligned}
$$


Thus we obtain the formula (1).

For example, we give an explicit formula for the colored Jones polynomial of the knot $6_{2}$, which is not a twist knot. The knot $6_{2}$ has the Schubert's normal form $S(11,7)$. We see $p^{\prime}=5$,

$$
\begin{aligned}
& \sigma_{1}=1, \sigma_{2}=-1, \sigma_{3}=-1, \sigma_{4}=1, \sigma_{5}=1, \\
& r^{\prime}(1)=4, r^{\prime}(2)=1, r^{\prime}(3)=5, r^{\prime}(4)=3, r^{\prime}(5)=2, i_{1}=2, i_{2}=5, i_{3}=4, i_{4}=1, i_{5}=3, \\
& \sigma_{i_{1}}=-1, \sigma_{i_{2}}=-1, \sigma_{i_{3}}=1, \sigma_{i_{4}}=1, \sigma_{i_{5}}=-1,
\end{aligned}
$$

and so

$$
\begin{aligned}
& \sum_{k=r^{\prime}(1)}^{5}\left(\sigma_{i_{k}}+\sigma_{i_{6-k}}\right)=-2, \sum_{k=r^{\prime}(2)}^{5}\left(\sigma_{i_{k}}+\sigma_{i_{6-k}}\right)=-2, \sum_{k=r^{\prime}(3)}^{5}\left(\sigma_{i_{k}}+\sigma_{i_{6-k}}\right)=-2, \\
& \sum_{k=r^{\prime}(4)}^{5}\left(\sigma_{i_{k}}+\sigma_{i_{6-k}}\right)=0, \sum_{k=r^{\prime}(5)}^{5}\left(\sigma_{i_{k}}+\sigma_{i_{6-k}}\right)=0, \\
& \sum_{j=1}^{4}\left(\sigma_{j+1}+\sigma_{6-j}\right)=-2 m_{1}-2 m_{4} .
\end{aligned}
$$

Thus, we get $a(\mathbf{m})=1+m_{1}+m_{3}+m_{4}$. Moreover, we calculate

$$
\begin{aligned}
& \sum_{1 \leq k \leq 2} \frac{1-\sigma_{i_{k}}}{2} m_{i_{k}-1}=m_{1}+m_{4}, \sum_{3 \leq k \leq 2} m_{i_{k}-1}=m_{3}+m_{2}, \sum_{3 \leq k \leq 5} \frac{1+\sigma_{i_{k}}}{2} m_{i_{k}}=m_{4}+m_{1}, \\
& 1+\sigma_{5}=0, \quad \sum_{j=1}^{4}\left(\sigma_{j+1}-\sigma_{j}\right) m_{j}=-2 m_{1}+2 m_{3}-2 m_{4}, \\
& \quad \sum_{1 \leq k \leq p^{\prime}-1} \sum_{k+1 \leq k^{\prime} \leq p^{\prime}} \frac{1+\operatorname{sign}\left(i_{k}-i_{k^{\prime}}\right)}{2}\left(\sigma_{i_{k}}-\sigma_{i_{k^{\prime}}}\right)\left(m_{i_{k}}-m_{i_{k}-1}\right)\left(m_{i_{k^{\prime}}}-m_{i_{k^{\prime}}-1}\right) \\
& =-2\left(m_{2}-m_{1}\right) m_{1}-2\left(m_{5}-m_{4}\right)\left(m_{4}-m_{3}\right)-2\left(m_{5}-m_{4}\right) m_{1}+2\left(m_{4}-m_{3}\right)\left(m_{3}-m_{2}\right), \\
& \sum_{j=1}^{p^{\prime}} \sigma_{j}\left(\sum_{k=1}^{r^{\prime}(j)}\left(m_{i_{k}}-m_{i_{k}-1}\right)\right) m_{j-1} \\
& \left.=-m_{1}\left(m_{2}-m_{1}\right)-m_{2} m_{5}\right)+m_{3}\left(m_{2}-m_{1}+m_{5}-m_{3}\right)+\left(-m_{4}\right)\left(m_{2}-m_{1}+m_{5}-m_{4}\right),
\end{aligned}
$$

and so $b_{1}(\mathbf{m})=-1-m_{2}-m_{3}+m_{1} m_{5}-m_{2} m_{5}-m_{1} m_{3}$. It is easy to compute $b_{2}(\mathbf{m})=m_{3}$. 
Finally, we obtain

$$
\begin{aligned}
= & \sum_{\substack{0 \leq m_{j-1} \leq m_{j} \leq n-1 \\
1 \leq j \leq 5}}^{J_{S(11,7)}(n)} q^{n\left(1+m_{1}+m_{3}+m_{4}\right)-1-m_{2}+m_{1} m_{5}-m_{2} m_{5}-m_{1} m_{3}} \\
& \times \frac{(q)_{n-1}(q)_{m_{5}}}{(q)_{n-m_{5}-1}}\left((\bar{q})_{m_{1}}(q)_{m_{2}-m_{1}}(q)_{m_{3}-m_{2}}(\bar{q})_{m_{4}-m_{3}}(q)_{m_{5}-m_{4}}\right)^{-1}(-1)^{m_{5}} .
\end{aligned}
$$

\section{Results for a twist knot}

In this section, we will consider the twist knots $S\left(4 p_{1}+1,2 p_{1}+1\right)$ and $S\left(4 p_{1}-\right.$ $\left.1,2 p_{1}-1\right)$.

From Theorem 1, we get explicit formulas for the colored Jones polynomials of these twist knots.

\section{Corollary 5.}

$$
\begin{aligned}
& J_{S\left(4 p_{1}+1,2 p_{1}+1\right)}(n) \\
& =\sum_{0 \leq m_{j-1} \leq m_{j} \leq n-1} q^{-n \sum_{i=1}^{2 p_{1}-1}(-1)^{i} m_{i}+\sum_{i=1}^{p_{1}-1} m_{2 i}\left(1+m_{2 i+1}\right)} \\
& \times \frac{(\bar{q})_{n-1}(\bar{q})_{m_{p^{\prime}}}}{(\bar{q})_{n-m_{p^{\prime}}-1}}(-1)^{m_{p^{\prime}}}\left(\prod_{j=0}^{p_{1}-1}(\bar{q})_{m_{2 j+1}-m_{2 j}} \prod_{j=1}^{p_{1}}(q)_{m_{2 j}-m_{2 j-1}}\right)^{-1},
\end{aligned}
$$

and

$$
\begin{aligned}
& =\sum_{0 \leq m_{j-1} \leq m_{j} \leq n-1} q_{S\left(4 p_{1}-1,2 p_{1}-1\right)}(n) \\
& \left.\quad \times \frac{(q)_{n-1}(q)_{m_{p^{\prime}}}}{(q)_{n-m_{p^{\prime}}-1}}(-1)^{m_{p^{\prime}}}\left(\prod_{j=0}^{p_{1}-1}(\bar{q})_{m_{2 j+1}-m_{2 j}} \prod_{j=1}^{p_{1}-1}(q)_{m_{2 j}-m_{2 j-1}}\right)^{-1}\right)^{-1} .
\end{aligned}
$$

Proof. Firstly, let us consider the $\operatorname{knot} S\left(4 p_{1}+1,2 p_{1}+1\right)$ for $p_{1} \geq 1$. It is easy to see that

Lemma 6.(1) $\sigma_{j}=(-1)^{j-1}$
(2) $i_{k}= \begin{cases}2 p_{1}+2-2 k & \text { if } 1 \leq k \leq p_{1} \\ 2\left(k-p_{1}\right)-1 & \text { if } p_{1}+1 \leq k \leq 2 p_{1}\end{cases}$
$\sigma_{i_{k}}= \begin{cases}-1 & \text { if } 1 \leq k \leq p_{1} \\ 1 & \text { if } p_{1}+1 \leq k \leq 2 p_{1}\end{cases}$ 
Lemma 7. (1) $\sigma_{i_{k}}+\sigma_{i_{p^{\prime}+1-k}}=0 . \quad$ (2) $\sigma_{j+1}+\sigma_{p^{\prime}+1-j}=(-2)^{j}$.

From the above lemmas, we obtain

$$
a(\mathbf{m})=-\sum_{j=1}^{2 p_{1}-1}(-1)^{j} m_{j}
$$

We compute $b_{1}(\mathbf{m})$ and $b_{2}(\mathbf{m})$. One calculate

$$
\sum_{1 \leq k \leq \frac{p-t}{2}} \frac{1-\sigma_{i_{k}}}{2} m_{i_{k}-1}=\sum_{j=1}^{p_{1}} m_{2 j-1}, \quad \sum_{\frac{p-t}{2}+1 \leq k \leq p^{\prime}} m_{i_{k}-1}=\sum_{j=1}^{p_{1}-1} m_{2 j}
$$

$$
\begin{aligned}
& \sum_{\frac{p-t}{2}+1 \leq k \leq p^{\prime}} \frac{1+\sigma_{i_{k}}}{2} m_{i_{k}} \\
= & \sum_{p_{1}+1 \leq k \leq 2 p_{1}} m_{i_{k}}=\sum_{p_{1}+1 \leq k \leq 2 p_{1}} m_{2 k-2 p_{1}-1}=\sum_{j=1}^{p_{1}} m_{2 j-1} \\
& \left(1+\sigma_{p^{\prime}}\right) m_{p^{\prime}}=0, \\
\text { (19) } \quad & \sum_{j=1}^{p^{\prime}-1}\left(\sigma_{j+1}-\sigma_{j}\right) m_{j}=-2 \sum_{j=1}^{p_{1}} m_{2 j-1}+2 \sum_{j=1}^{p_{1}-1} m_{2 j}=2 \sum_{j=1}^{2 p_{1}-1}(-1)^{j} m_{j} .
\end{aligned}
$$

Moreover, it follows that $k<k^{\prime}$ and $\sigma_{i_{k}} \neq \sigma_{i_{k^{\prime}}}$ if and only if $1 \leq k \leq p_{1}$ and $p_{1}+1 \leq k^{\prime} \leq 2 p_{1}$. We note that $\operatorname{sign}\left(i_{k}-i_{k^{\prime}}\right)=1$ if and only if $i_{k}>i_{k^{\prime}}$ and that $i_{k}=2 p_{1}+2-2 k$ for $1 \leq k \leq p_{1}$ and $i_{k^{\prime}}=2 k^{\prime}-2 p_{1}-1$ for $p_{1}+1 \leq k^{\prime} \leq 2 p_{1}$.

So, we obtain

$$
\begin{aligned}
& \sum_{1 \leq k \leq p^{\prime}} \sum_{k+1 \leq k^{\prime} \leq p^{\prime}} \frac{1+\operatorname{sign}\left(i_{k}-i_{k^{\prime}}\right)}{2}\left(\sigma_{i_{k}}-\sigma_{i_{k^{\prime}}}\right)\left(m_{i_{k}}-m_{i_{k}-1}\right)\left(m_{i_{k^{\prime}}}-m_{i_{k^{\prime}}-1}\right) \\
= & -2 \sum_{1 \leq k \leq p_{1}} \sum_{p_{1}+1 \leq k^{\prime} \leq 2 p_{1}+1-k}\left(m_{2 p_{1}+2-2 k}-m_{2 p_{1}+1-2 k}\right)\left(m_{2 k^{\prime}-2 p_{1}-1}-m_{2 k^{\prime}-2 p_{1}-2}\right) \\
= & -2 \sum_{1 \leq j \leq p_{1}} \sum_{1 \leq j^{\prime} \leq j}\left(m_{2 j}-m_{2 j-1}\right)\left(m_{2 j^{\prime}-1}-m_{2 j^{\prime}-2}\right),
\end{aligned}
$$


and

$$
\begin{aligned}
& \sum_{j=1}^{p^{\prime}} \sigma_{j}\left(\sum_{k=1}^{r^{\prime}(j)}\left(m_{i_{k}}-m_{i_{k}-1}\right)\right) m_{j-1} \\
= & \sum_{j=1}^{p_{1}} \sigma_{2 j-1}\left(\sum_{k=1}^{r^{\prime}(2 j-1)}\left(m_{i_{k}}-m_{i_{k}-1}\right)\right) m_{2 j-2}+\sum_{j=1}^{p_{1}} \sigma_{2 j}\left(\sum_{k=1}^{r^{\prime}(2 j)}\left(m_{i_{k}}-m_{i_{k}-1}\right)\right) m_{2 j-1} \\
= & \sum_{j=1}^{p_{1}} \sigma_{2 j-1}\left(\sum_{k=1}^{p_{1}+j}\left(m_{i_{k}}-m_{i_{k}-1}\right)\right) m_{2 j-2}+\sum_{j=1}^{p_{1}} \sigma_{2 j}\left(\sum_{k=1}^{p_{1}-j+1}\left(m_{i_{k}}-m_{i_{k}-1}\right)\right) m_{2 j-1} \\
= & \sum_{j=1}^{p_{1}}\left(\sum_{k=1}^{p_{1}}\left(m_{2 p_{1}+2-2 k}-m_{2 p_{1}+1-2 k}\right)+\sum_{k=p_{1}+1}^{p_{1}+j}\left(m_{2 k-2 p_{1}-1}-m_{2 k-2 p_{1}-2}\right)\right) m_{2 j-2} \\
& -\sum_{j=1}^{p_{1}}\left(\sum_{k=1}^{p_{1}-j+1}\left(m_{2 p_{1}+2-2 k}-m_{2 p_{1}+1-2 k}\right)\right) m_{2 j-1} \\
= & \sum_{j=1}^{p_{1}}\left(\sum_{j^{\prime}=1}^{p_{1}}\left(m_{2 j^{\prime}}-m_{2 j^{\prime}-1}\right)+\sum_{j^{\prime}=1}^{j}\left(m_{2 j^{\prime}-1}-m_{2 j^{\prime}-2}\right)\right) m_{2 j-2} \\
& -\sum_{j=1}^{p_{1}}\left(\sum_{j^{\prime}=j}^{p_{1}}\left(m_{2 j^{\prime}}-m_{2 j^{\prime}-1}\right)\right) m_{2 j-1} \\
= & \sum_{j=1}^{p_{1}}\left(m_{2 j}+\sum_{j^{\prime}=j+1}^{p_{1}}\left(m_{2 j^{\prime}}-m_{2 j^{\prime}-1}\right)\right) m_{2 j-2}-\sum_{j=1}^{p_{1}}\left(\sum_{j^{\prime}=j}^{p_{1}}\left(m_{2 j^{\prime}}-m_{2 j^{\prime}-1}\right)\right) m_{2 j-1} \\
= & \sum_{j=1}^{p_{1}}\left(\sum_{j^{\prime}=j}^{p_{1}}\left(m_{2 j^{\prime}}-m_{2 j^{\prime}-1}\right)+m_{2 j-1}\right) m_{2 j-2}-\sum_{j=1}^{p_{1}}\left(\sum_{j^{\prime}=j}^{p_{1}}\left(m_{2 j^{\prime}}-m_{2 j^{\prime}-1}\right)\right) m_{2 j-1} .
\end{aligned}
$$

Summing up, we obtain

$$
b_{1}(\mathbf{m})+b_{2}(\mathbf{m})=\sum_{j=1}^{p_{1}-1} m_{2 j}\left(1+m_{2 j+1}\right) .
$$

Next, we consider the knot $S\left(4 p_{1}-1,2 p_{1}-1\right)$ for $p_{1} \geq 1$. It is not hard to show the following two lemmas.

Lemma 8. It follows that (1) $\sigma_{j}=(-1)^{j-1}$, and
(2) $i_{k}= \begin{cases}2 p_{1}-2 k+1 & \text { if } 1 \leq k \leq p_{1} \\ 2\left(k-p_{1}\right) & \text { if } p_{1}+1 \leq k \leq 2 p_{1}-1\end{cases}$
$\sigma_{i_{k}}= \begin{cases}1 & \text { if } 1 \leq k \leq p_{1} \\ -1 & \text { if } p_{1}+1 \leq k \leq 2 p_{1}-1\end{cases}$

Lemma 9. (1) $\sigma_{i_{k}}+\sigma_{i_{p^{\prime}+1-k}}=\left\{\begin{array}{ll}2 & \text { if } k=p_{1}, \\ 0 & \text { if } k \neq p_{1} .\end{array} \quad\right.$ (2) $\sigma_{j+1}+\sigma_{p^{\prime}+1-j}=0$. 
From these lemmas, it follows that

$$
a(\mathbf{m})=\sum_{i=1}^{2 p_{1}-2}(-1)^{i} m_{i}-2 m_{2 p_{1}-1}-1,
$$

We compute

$$
\begin{aligned}
& \sum_{1 \leq k \leq \frac{p-t}{2}} \frac{1-\sigma_{i_{k}}}{2} m_{i_{k}-1}=\sum_{1 \leq k \leq p_{1}} \frac{1-\sigma_{i_{k}}}{2} m_{i_{k}-1}=0, \\
& \sum_{\substack{\frac{p-t}{2}+1 \leq k \leq p^{\prime} \\
p_{1}-1}} m_{i_{k}-1}=\sum_{p_{1}+1 \leq k \leq 2 p_{1}-1} m_{i_{k}-1}=\sum_{p_{1}+1 \leq k \leq 2 p_{1}-1} m_{2 k-2 p_{1}-1} \\
& =\sum_{j=1} m_{2 j-1}, \\
& \sum_{\frac{p-t}{2}+1 \leq k \leq p^{\prime}} \frac{1+\sigma_{i_{k}}}{2} m_{i_{k}}=\sum_{p_{1}+1 \leq k \leq 2 p_{1}-1} \frac{1+\sigma_{i_{k}}}{2} m_{i_{k}}=0, \\
& \left(1+\sigma_{p^{\prime}}\right) m_{p^{\prime}}=2 m_{2 p_{1}-1}, \\
& \sum_{j=1}^{p^{\prime}-1}\left(\sigma_{j+1}-\sigma_{j}\right) m_{j}=\sum_{j=1}^{2 p_{1}-2}\left(\sigma_{j+1}-\sigma_{j}\right) m_{j}=2 \sum_{i=1}^{2 p_{1}-2}(-1)^{i} m_{i} .
\end{aligned}
$$

Moreover, it follows that $k<k^{\prime}$ and $\sigma_{i_{k}} \neq \sigma_{i_{k^{\prime}}}$ if and only if $1 \leq k \leq p_{1}$ and $p_{1}+1 \leq k^{\prime} \leq 2 p_{1}-1$. We note that $\operatorname{sign}\left(i_{k}-i_{k^{\prime}}\right)=1$ if and only if $i_{k}>i_{k^{\prime}}$ and that $i_{k}=2 p_{1}-2 k+1$ for $1 \leq k \leq p_{1}$ and $i_{k^{\prime}}=2 k^{\prime}-2 p_{1}$ for $p_{1}+1 \leq k^{\prime} \leq 2 p_{1}-1$.

So, we compute

$$
\begin{aligned}
& \sum_{1 \leq k \leq p^{\prime}} \sum_{k+1 \leq k^{\prime} \leq p^{\prime}} \frac{1+\operatorname{sign}\left(i_{k}-i_{k^{\prime}}\right)}{2}\left(\sigma_{i_{k}}-\sigma_{i_{k^{\prime}}}\right)\left(m_{i_{k}}-m_{i_{k}-1}\right)\left(m_{i_{k^{\prime}}}-m_{i_{k^{\prime}}-1}\right) \\
= & 2 \sum_{1 \leq k \leq p_{1}} \sum_{p_{1}+1 \leq k^{\prime} \leq 2 p_{1}-k}\left(m_{2 p_{1}-2 k+1}-m_{2 p_{1}-2 k}\right)\left(m_{2 k^{\prime}-2 p_{1}}-m_{2 k^{\prime}-2 p_{1}-1}\right) \\
= & 2 \sum_{0 \leq j \leq p_{1}-1} \sum_{1 \leq j^{\prime} \leq j}\left(m_{2 j+1}-m_{2 j}\right)\left(m_{2 j^{\prime}}-m_{2 j^{\prime}-1}\right),
\end{aligned}
$$


and

$$
\begin{aligned}
& \sum_{j=1}^{p^{\prime}} \sigma_{j}\left(\sum_{k=1}^{r^{\prime}(j)}\left(m_{i_{k}}-m_{i_{k}-1}\right)\right) m_{j-1} \\
& =\sum_{j=1}^{p_{1}} \sigma_{2 j-1}\left(\sum_{k=1}^{r^{\prime}(2 j-1)}\left(m_{i_{k}}-m_{i_{k}-1}\right)\right) m_{2 j-2}+\sum_{j=1}^{p_{1}-1} \sigma_{2 j}\left(\sum_{k=1}^{r^{\prime}(2 j)}\left(m_{i_{k}}-m_{i_{k}-1}\right)\right) m_{2 j-1} \\
& =\sum_{j=1}^{p_{1}}\left(\sum_{k=1}^{p_{1}-j+1}\left(m_{i_{k}}-m_{i_{k}-1}\right)\right) m_{2 j-2}-\sum_{j=1}^{p_{1}-1}\left(\sum_{k=1}^{p_{1}-j}\left(m_{i_{k}}-m_{i_{k}-1}\right)\right) m_{2 j-1} \\
& =\sum_{j=1}^{p_{1}}\left(\sum_{k=1}^{p_{1}-j+1}\left(m_{2 p_{1}-2 k+1}-m_{2 p_{1}-2 k}\right)\right) m_{2 j-2} \\
& -\sum_{j=1}^{p_{1}-1}\left(\sum_{k=1}^{p_{1}}\left(m_{2 k-2 p_{1}+1}-m_{2 k-2 p_{1}}\right)+\sum_{k=p_{1}+1}^{p_{1}+j}\left(m_{2 p_{1}-2 k}-m_{2 p_{1}-2 k-1}\right)\right) m_{2 j-1} \\
& =\sum_{j=1}^{p_{1}}\left(\sum_{j^{\prime}=j}^{p_{1}}\left(m_{2 j^{\prime}-1}-m_{2 j^{\prime}-2}\right) m_{2 j-2}-\sum_{j=1}^{p_{1}-1}\left(\sum_{j^{\prime}=1}^{p_{1}-1}\left(m_{2 j^{\prime}+1}-m_{2 j^{\prime}}\right)+\sum_{j^{\prime}=1}^{j}\left(m_{2 j^{\prime}}-m_{2 j^{\prime}-1}\right)\right) m_{2 j-1}\right. \\
& =\sum_{j=1}^{p_{1}}\left(\sum_{j^{\prime}=j}^{p_{1}}\left(m_{2 j^{\prime}-1}-m_{2 j^{\prime}-2}\right)\right) m_{2 j-2}-\sum_{j=1}^{p_{1}-1}\left(\sum_{j^{\prime}=j+1}^{p_{1}}\left(m_{2 j^{\prime}-1}-m_{2 j^{\prime}-2}\right)+m_{2 j}\right) m_{2 j-1} \\
& =\sum_{0 \leq j^{\prime} \leq p_{1}-1} \sum_{1 \leq j \leq j^{\prime}}\left(m_{2 j^{\prime}+1}-m_{2 j^{\prime}}\right)\left(m_{2 j}-m_{2 j-1}\right)-\sum_{j=1}^{p_{1}-1} m_{2 j} m_{2 j-1} \text {. }
\end{aligned}
$$

Thus we obtain

$$
b_{1}(\mathbf{m})+b_{2}(\mathbf{m})=1-\sum_{j=1}^{p_{1}-1} m_{2 j-1}\left(1+m_{2 j}\right) .
$$

Using Corollary 5, we give explicit formulas for the trefoil knot $S(3,1)$ and the figure eight knot $S(5,3)$.

By the formula (15) with $p_{1}=1$ and $p^{\prime}=1$, we get

$$
\begin{aligned}
J_{S(3,1)}(n) & =\sum_{0 \leq m_{1} \leq n-1} \frac{(q)_{n-1}(q)_{m_{1}}(-1)^{m_{1}}}{(q)_{n-m_{1}-1}(\bar{q})_{m_{1}}} q^{n\left(-1-2 m_{1}\right)+1} \\
& =\sum_{0 \leq m_{1} \leq n-1}\left(\bar{q}^{n-1}-1\right) \cdots\left(\bar{q}^{n-m_{1}}-1\right)(-1)^{m_{1}} q^{n\left(-1-m_{1}\right)+1}
\end{aligned}
$$


By the formula (14) with $p_{1}=1$ and $p^{\prime}=1$,

$$
J_{S(5,3)}(n)=\sum_{0 \leq m_{1} \leq m_{2} \leq n-1} \frac{(\bar{q})_{n-1}(\bar{q})_{m_{2}}}{(\bar{q})_{n-m_{2}-1}(\bar{q})_{m_{1}}(q)_{m_{2}-m_{1}}} q^{n m_{1}}(-1)^{m_{2}}
$$

and using the formula

$$
\sum_{i=0}^{k} \frac{(q)_{k}}{(q)_{k-i}(\bar{q})_{i}} q^{-\frac{i}{2}(k+b+1)}=\prod_{j=1}^{k}\left(1-q^{-\frac{1}{2}(k+b+1-2 j)}\right),
$$

we obtain a formula of K. Habiro [2] or T. T. Q. Le as follows,

$$
J_{S(5,3)}(n)=\sum_{0 \leq m \leq n-1} \frac{(q)_{n-1}(q)_{n+m}}{(q)_{n}(q)_{m}(q)_{n-m-1}} q^{-n m} .
$$

Finally, as an application of Corollary 5 , we estimate the maximal and minimal degree of $J_{K}(n)$ for a twist knot $K$. We denote by $\operatorname{deg}_{+}\left(J_{K}(n)\right)$ and $\operatorname{deg}_{-}\left(J_{K}(n)\right)$ the maximal degree and the minimal degree in $q$ of $J_{K}(n) \in \mathbb{Z}\left[q^{ \pm 1}\right]$ respectively, and let $\operatorname{br}\left(J_{K}(n)\right)=\operatorname{deg}_{+}\left(J_{K}(n)\right)-\operatorname{deg}_{-}\left(J_{K}(n)\right)$. From Corollary 5, we obtain

Corollary 10. For $K=S\left(4 p_{1}+1,2 p_{1}+1\right)$,

$$
\begin{aligned}
& \operatorname{deg}_{+}\left(J_{K}(n)\right)=\frac{1}{2} n(n-1)\left(2 p_{1}\right), \quad \operatorname{deg}_{-}\left(J_{K}(n)\right)=-n(n-1), \\
& \operatorname{br}\left(J_{K}(n)\right)=\frac{1}{2} n(n-1)\left(2 p_{1}+2\right),
\end{aligned}
$$

and for $K=S\left(4 p_{1}-1,2 p_{1}-1\right)$,

(30) $\operatorname{deg}_{+}\left(J_{K}(n)\right)=-n+1, \quad \operatorname{deg}_{-}\left(J_{K}(n)\right)=-n^{2}+1-\frac{1}{2} n(n-1)\left(2 p_{1}-1\right)$,

$$
\operatorname{br}\left(J_{K}(n)\right)=\frac{1}{2} n(n-1)\left(2 p_{1}+1\right) .
$$

Thus, it follows that for $K=S\left(4 p_{1}+1,2 p_{1}+1\right)$ or $K=S\left(4 p_{1}-1,2 p_{1}-1\right)$,

$$
\operatorname{br}\left(J_{K}(n)\right)=\frac{1}{2} n(n-1) C(K),
$$

where $C(K)$ is the crossing number of $K$.

Proof. From Corollary 5, we obtain

$$
\begin{aligned}
J_{S\left(4 p_{1}+1,2 p_{1}+1\right)}(n)= & \sum_{0 \leq m_{j-1} \leq m_{j} \leq n-1} q^{-n \sum_{i=1}^{2 p_{1}}(-1)^{i} m_{i}+\frac{1}{2} \sum_{i=1}^{2 p_{1}-1} m_{i}\left(1+m_{i}\right)}(-1)^{\sum_{i=1}^{2 p_{1}} m_{i}} \\
& \times\left(q^{n-1}-1\right) \cdots\left(q^{n-m_{p^{\prime}}}-1\right) \prod_{j=2}^{2 p_{1}} \frac{(q)_{m_{j}}}{(q)_{m_{j-1}}(q)_{m_{j}-m_{j-1}}}
\end{aligned}
$$


Notice that the maximal degree in $q$ of $\frac{(q)_{a}}{(q)_{b}(q)_{a-b}}$ is $b(a-b)$ and the minimal degree is 1 . Noting that

$$
\begin{aligned}
& -n(n-1) \\
\leq & n\left(m_{1}+\sum_{i=1}^{p_{1}-1}\left(m_{2 i+1}-m_{2 i}\right)-m_{2 p_{1}}\right)+\frac{1}{2} \sum_{i=1}^{2 p_{1}-1} m_{i}\left(1+m_{i}\right)+\sum_{j=2}^{2 p_{1}} m_{j-1}\left(m_{j}-m_{j-1}\right) \\
= & -n \sum_{i=1}^{p_{1}-1} l_{2 i}-\frac{1}{2} \sum_{i=1}^{2 p_{1}-1}\left(m_{i}-m_{i+1}\right)^{2}+\frac{1}{2} \sum_{i=1}^{2 p_{1}-1} m_{i+1}^{2}+\frac{1}{2} \sum_{i=1}^{2 p_{1}-1} m_{i} \\
\leq & \frac{1}{2} n(n-1)\left(2 p_{1}-1\right),
\end{aligned}
$$

and

$$
0 \leq \frac{1}{2} m_{2 p_{1}}\left(2 n-1-m_{2 p_{1}}\right) \leq \frac{1}{2} n(n-1),
$$

it follows that the term with $m_{i}=n-1$ contributes to $\operatorname{deg}_{+}\left(J_{K}(n)\right)$ and the term with $m_{i}=0\left(i \neq p^{\prime}\right)$ and $m_{p^{\prime}}=n-1$ to $\operatorname{deg}_{-}\left(J_{K}(n)\right)$. So, the formula (29) holds. Similarly, from Corollary 5 ,

$$
\begin{aligned}
J_{S\left(4 p_{1}-1,2 p_{1}-1\right)}(n)= & \sum_{0 \leq m_{j-1} \leq m_{j} \leq n-1} q^{n\left(-1+\sum_{i=1}^{2 p_{1}-1}(-1)^{i} m_{i}\right)+1-\frac{1}{2} \sum_{i=1}^{2 p_{1}-2} m_{i}\left(1+m_{i}\right)}(-1)^{\sum_{i=1}^{2 p_{1}-1} m_{i}} \\
& \times\left(\bar{q}^{n-1}-1\right) \cdots\left(\bar{q}^{n-m_{p^{\prime}}}-1\right) \prod_{j=2}^{2 p_{1}-1} \frac{(\bar{q})_{m_{j}}}{(\bar{q})_{m_{j-1}}(\bar{q})_{m_{j}-m_{j-1}}} .
\end{aligned}
$$

Since

$$
\begin{aligned}
& -n+1 \\
\geq & n\left(-1-m_{1}-\sum_{i=1}^{p_{1}-1}\left(m_{2 i+1}-m_{2 i}\right)\right)+1-\frac{1}{2} \sum_{i=1}^{2 p_{1}-2} m_{i}\left(1+m_{i+1}\right)-\sum_{j=2}^{2 p_{1}-1} m_{j-1}\left(m_{j}-m_{j-1}\right) \\
= & n\left(-1-\sum_{i=1}^{p_{1}} l_{2 i-1}+1\right)+\frac{1}{2} \sum_{i=1}^{2 p_{1}-2}\left(m_{i}-m_{i-1}\right)^{2}-\frac{1}{2} \sum_{i=1}^{2 p_{1}-2} m_{i+1}^{2}-\frac{1}{2} \sum_{i=1}^{2 p_{1}-2} m_{i} \\
\geq & -n^{2}+1-\frac{1}{2} n(n-1)\left(2 p_{1}-2\right),
\end{aligned}
$$

and

$$
0 \geq-\frac{1}{2}\left(2 n-1-m_{2 p_{1}+1}\right) m_{2 p_{1}+1} \geq-\frac{1}{2} n(n-1),
$$

the term with $m_{i}=0$ contributes to $\operatorname{deg}_{+}\left(J_{K}(n)\right)$ and the term with $m_{i}=n-1$ $\left(i \neq p^{\prime}\right)$ and $m_{p^{\prime}}=n-1$ to $\operatorname{deg}_{-}\left(J_{K}(n)\right)$. Thus, we get the formula (30).

Moreover, since $S\left(4 p_{1}+1,2 p_{1}+1\right)$ (resp. $S\left(4 p_{1}-1,2 p_{1}-1\right)$ ) is given in the Conway notation by $C\left(2 p_{1}, 2\right)$ (resp. $\left.C\left(2 p_{1}-1,2\right)\right)$, the equality (31) holds. 
Remark 11. Recently, Le proved that the equality (31) holds for any non-trivial alternating knot (see Proposition 2.1 of [6]).

Acknowledgment. We would like to thank the referee for many helpful comments.

\section{References}

[1] G. Burde and H. Zieschang, Knots, Walter de Gruyter \& Co.,Berlin, 1985.

[2] K. Habiro, On the quantum sl $_{2}$ invariants of knots and integral homology spheres, Geometry and Topology Monographs, 4(5)(2000), 55-68.

[3] V. F. R. Jones, A polynomial invariants for knots via von Neumann algebras, Bull. Amer. Math. Soc., N.S.(1985), 103-111.

[4] A. Kawauchi, A survey of Knot Theory, Birkhauser Verlag, Switzerland, 1996

[5] R. Lawrence and O. Ron, On Habiro's cyclotomic expansions of Ohtsuki invariant, (math.GT/0501549).

[6] T. T. Q. Le, The colored Jones polynomial and A-polynomial of two-bridge knots, (math.GT/0407521).

[7] G. Masbaum, Skein-theoretical derivation of some formulas of Habiro, Algebraic and Geomteric Topology, 3(17)(2003), 537-556. 